\title{
1 A Hardware Abstraction Layer in Java
}

2 MARTIN SCHOEBERL, Vienna University of Technology

3 STEPHAN KORSHOLM, Aalborg University

4 TOMAS KALIBERA, Purdue University

5 ANDERS P. RAVN, Aalborg University

6 Embedded systems use specialized hardware devices to interact with their environment, and since they

7 have to be dependable, it is attractive to use a modern, type-safe programming language like Java to de-

8 velop programs for them. Standard Java, as a platform-independent language, delegates access to devices,

9 direct memory access, and interrupt handling to some underlying operating system or kernel, but in the

10 embedded systems domain resources are scarce and a Java Virtual Machine (JVM) without an underlying

11 middleware is an attractive architecture. The contribution of this article is a proposal for Java packages

12 with hardware objects and interrupt handlers that interface to such a JVM. We provide implementations

13 of the proposal directly in hardware, as extensions of standard interpreters, and finally with an operating

14 system middleware. The latter solution is mainly seen as a migration path allowing Java programs to co-

15 exist with legacy system components. An important aspect of the proposal is that it is compatible with the

16 Real-Time Specification for Java (RTSJ).

17 Categories and Subject Descriptors: D.4.7 [Operating Systems]: Organization and Design-Real-time 18 systems and embedded systems; D.3.3 [Programming Languages]: Language Constructs and Features19 Input/output

20 General Terms: Languages, Design

21 Additional Key Words and Phrases: Device driver, embedded system, Java, Java virtual machine

22 ACM Reference Format:

23 Schoeberl, M., Korsholm, S., Kalibera, T., and Ravn, A. P. 2011. A hardware abstraction layer in Java. ACM

24 Trans. Embed. Comput. Syst. 10, 4, Article 42 (November 2011), 40 pages.

25 DOI $=10.1145 / 2043662.2043666$ http://doi.acm.org/10.1145/2043662.2043666

\section{1. INTRODUCTION}

27 When developing software for an embedded system, for instance an instrument, it is 28 necessary to control specialized hardware devices, for instance a heating element or an 29 interferometer mirror. These devices are typically interfaced to the processor through 30 device registers and may use interrupts to synchronize with the processor. In order 31 to make the programs easier to understand, it is convenient to introduce a Hardware 32 Abstraction Layer (HAL), where access to device registers and synchronization through 33 interrupts are hidden from conventional program components. A HAL defines an in34 terface in terms of the constructs of the programming language used to develop the

The research leading to these results received funding from the European Community's Seventh Framework Programme [FP7/2007-2013] under grant agreement number 216682 (JEOPARD).

Authors' addresses: M. Schoeberl (corresponding author), Vienna University of Technology, Austria; email: mschoebe@mail.tuwien.ac.at; S. Korsholm, Aalborg University, Denmark; T. Kalibera, Purdue University, U.S.A.; A. P. Ravn, Aalborg University, Denmark.

Permission to make digital or hard copies of part or all of this work for personal or classroom use is granted without fee provided that copies are not made or distributed for profit or commercial advantage and that copies show this notice on the first page or initial screen of a display along with the full citation. Copyrights for components of this work owned by others than ACM must be honored. Abstracting with credit is permitted. To copy otherwise, to republish, to post on servers, to redistribute to lists, or to use any component of this work in other works requires prior specific permission and/or a fee. Permissions may be requested from the Publications Dept., ACM, Inc., 2 Penn Plaza, Suite 701, New York, NY 10121-0701 USA, fax +1 (212) 869-0481, or permissions@acm.org.

(C) 2011 ACM 1539-9087/2011/11-ART42 $\$ 10.00$

DOI 10.1145/2043662.2043666 http://doi.acm.org/10.1145/2043662.2043666 
application. Thus, the challenge is to develop an abstraction that gives efficient access to the hardware, while staying within the computational model provided by the programming language.

Our first ideas on a HAL for Java have been published in Schoeberl et al. [2008] and Korsholm et al. [2008]. This article combines the two papers, provides a much wider background of related work, gives two additional experimental implementations, and gives performance measurements that allow an assessment of the efficiency of the implementations. The remainder of this section introduces the concepts of the Javabased HAL.

\subsection{Java for Embedded Systems}

Over the nearly 15 years of its existence Java has become a popular programming language for desktop and server applications. The concept of the Java Virtual Machine (JVM) as the execution platform enables portability of Java applications. The language, its API specification, as well as JVM implementations have matured; Java is today employed in large-scale industrial applications. The automatic memory management takes away a burden from the application programmers and together with type safety helps to isolate problems and, to some extent, even run untrusted code. It also enhances security; attacks like stack overflow are not possible. Java integrates threading support and dynamic loading into the language, making these features easily accessible on different platforms. The Java language and JVM specifications are proven by different implementations on different platforms, making it relatively easy to write platform-independent Java programs that run on different JVM implementations and underlying OS/hardware. Java has a standard API for a wide range of libraries, the use of which is thus again platform-independent. With the ubiquity of Java, it is easy to find qualified programmers who know the language, and there is strong tool support for the whole development process. According to an experimental study [Phipps 1999], Java has lower bug rates and higher productivity rates than C++. Indeed, some of these features come at a price of larger footprint (the virtual machine is a nontrivial piece of code), typically higher memory requirements, and sometimes degraded performance, but this cost is accepted in industry.

Recent real-time Java virtual machines based on the Real-Time Specification for Java (RTSJ) provide controlled and safe memory allocation. Also there are platforms for less critical systems with real-time garbage collectors. Thus, Java is ready to make its way into the embedded systems domain. Mobile phones, PDAs, or set-top boxes run Java Micro Edition, a Java platform with a restricted set of standard Java libraries. Real-time Java has been and is being evaluated as a potential future platform for space avionics both by NASA and ESA space agencies. Some Java features are even more important for embedded than for desktop systems because of missing features of the underlying platform. For instance, the RTEMS operating system used by ESA for space missions does not support hardware memory protection even for CPUs that do support it (like LEON3, a CPU for ESA space missions). With Java's type safety hardware protection is not needed to spatially isolate applications. Moreover, RTEMS does not support dynamic libraries, but Java can load classes dynamically.

Many embedded applications require very small platforms, therefore it is interesting to remove as much as possible of an underlying operating system or kernel, where a major part of code is dedicated to handling devices. Furthermore, Java is considered as the future language for safety-critical systems [Henties et al. 2009]. As certification of safety-critical systems is very expensive, the usual approach is to minimize the code base and supporting tools. Using two languages (e.g., C for programming device handling in the kernel and Java for implementing the processing of data) increases 


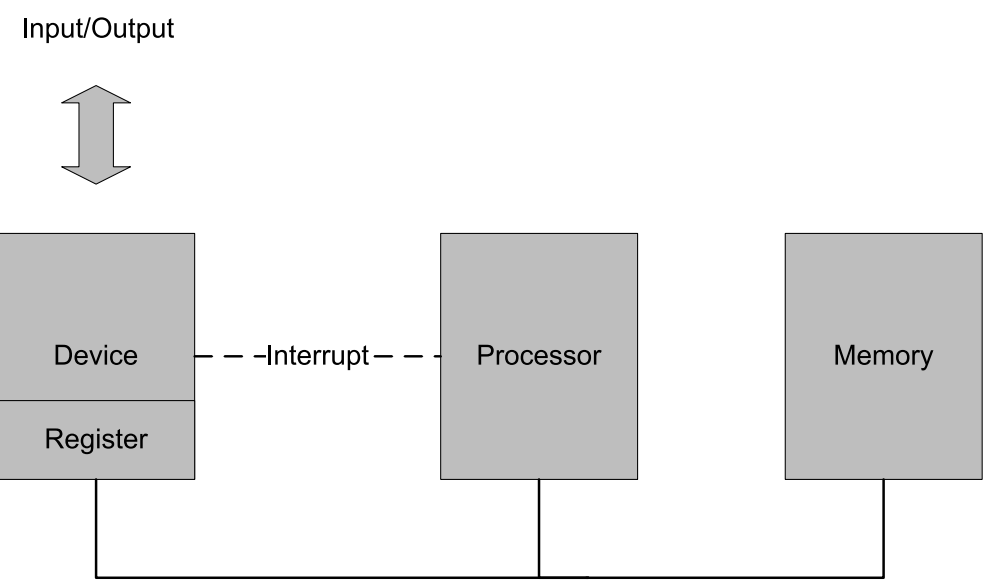

Fig. 1. The hardware: a bus connects a processor to device registers and memory, and an interrupt bus connects devices to a processor.

85 the complexity of generating a safety case. A Java-only system reduces the complexity 86 of the tool support and therefore the certification effort. Even in less critical systems 87 the same issues will show up as decreased productivity and dependability of the soft88 ware. Thus it makes sense to investigate a general solution that interfaces Java to the 89 hardware platform; that is the objective of the work presented here.

\section{1.2 Hardware Assumptions}

91 The hardware platform is built up along one or more buses (in small systems typically 92 only one) that connect the processor with memory and device controllers. Device con93 trollers have reserved some part of the address space of a bus for its device registers. 94 They are accessible for the processor as well, either through special I/O instructions or 95 by ordinary instructions when the address space is the same as the one for addressing 96 memory, a so called memory mapped I/O solution. In some cases the device controller 97 will have Direct Memory Access (DMA) as well, for instance for high-speed transfer of 98 blocks of data. Thus the basic communication paradigm between a controller and the 99 processor is shared memory through the device registers and/or through DMA. With 100 these facilities only, synchronization has to be done by testing and setting flags, which 101 means that the processor has to engage in some form of busy waiting. This is elim102 inated by extending the system with an interrupt bus, where device controllers can 103 generate a signal that interrupts the normal flow of execution in the processor and di104 rect it to an interrupt handling program. Since communication is through shared data 105 structures, the processor and the controllers need a locking mechanism; therefore in106 terrupts can be enabled or disabled by the processor through an interrupt control unit. 107 The typical hardware organization is summarized in Figure 1.

\section{1.3 A Computational Model}

109 In order to develop a HAL, the device registers and interrupt facilities must be mapped 110 to programming language constructs, such that their use corresponds to the computa111 tional model underlying the language. In the following we give simple device examples 112 which illustrate the solution we propose for doing it for Java.

113 1.3.1 Hardware Objects. Consider a simple Parallel Input/Output (PIO) device control114 ling a set of input and output pins. The PIO uses two registers: the data register and 


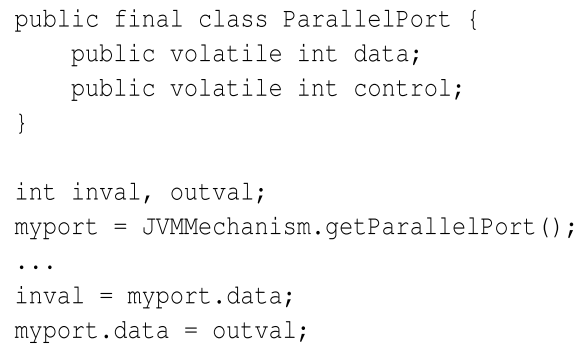

Fig. 2. The parallel port device as a simple Java class.

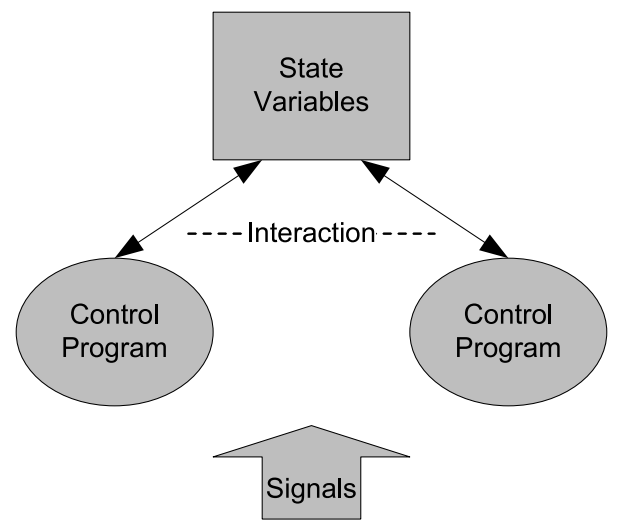

Fig. 3. Computational model: several threads of execution communicate via shared state variables and receive signals.

115 the control register. Writing to the data register stores the value into an internal latch 116 that drives the output pins. Reading from the data register returns the value that is 117 present on the input pins. The control register configures the direction for each PIO 118 pin. When bit $n$ in the control register is set to 1 , pin $n$ drives out the value of bit $n$ of 119 the data register. A 0 at bit $n$ in the control register configures pin $n$ as input pin. At 120 reset the port is usually configured as input port, a safe default configuration.

121 In an object-oriented language the most natural way to represent a device is as an 122 object: the hardware object. Figure 2 shows a class definition, object instantiation, 123 and use of the hardware object for the simple parallel port. An instance of the class 124 ParallelPort is the hardware object that represents the PIO. The reference myport points 125 to the hardware object. To provide this convenient representation of devices as objects, 126 a JVM internal mechanism is needed to access the device registers via object fields 127 and to create the device object and receive a reference to it. We elaborate on the idea of hardware objects in Section 3.1 and present implementations in Section 4.

129 1.3.2 Interrupts. When we consider an interrupt, it must invoke some program code 130 in a method that handles it. We need to map the interruption of normal execution to 131 some language concept, and here the concept of an asynchronous event is useful. The 132 resulting computational model for the programmer is shown in Figure 3. The signals 133 are external, asynchronous events that map to interrupts.

134 A layered implementation of this model with a kernel close to the hardware and 135 applications on top has been very useful in general-purpose programming. Here one

136 may even extend the kernel to manage resources and provide protection mechanisms 137 such that applications are safe from one another, as for instance when implementing 


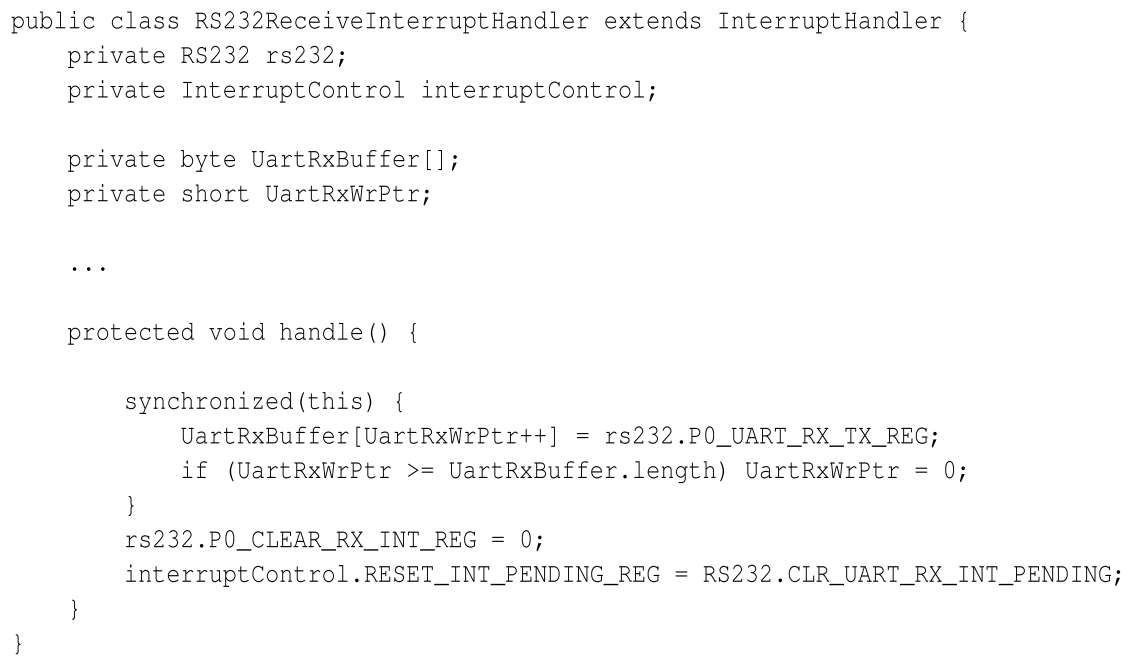

Fig. 4. An example interrupt handler for an RS232 interface. On an interrupt the method handle() is invoked. The private objects rs232 and interruptControl are hardware objects that represent the device registers and the interrupt control unit.

138 trusted interoperable computing platforms [Group 2008]. Yet there is a price to pay 139 which may make the solution less suitable for embedded systems: adding new device 140 drivers is an error-prone activity [Chou et al. 2001], and protection mechanisms impose 141 a heavy overhead on context switching when accessing devices.

142 The alternative we propose is to use Java directly since it already supports multi143 threading and use methods in the special InterruptHandler objects to handle interrupts. 144 The idea is illustrated in Figure 4, and the details, including synchronization and in145 teraction with the interrupt control, are elaborated in Section 3.2. Implementations 146 are found in Section 4.

\section{Mapping Between Java and the Hardware}

148 The proposed interfacing from hardware to Java does not require language extensions. 149 The Java concepts of packages, classes, and synchronized objects turn out to be power150 ful enough to formulate the desired abstractions. The mapping is done at the level of 151 the JVM. The JVM already provides typical OS functions handling:

152 - address space and memory management;

153 - thread management;

154 - interprocess communication.

155 These parts need to be modified so they cater for interfaces to the hardware.

156 Yet, the architectures of JVMs for embedded systems are more diverse than on 157 desktop or server systems. Figure 5 shows variations of Java implementations in 158 embedded systems and an example of the control flow for a Web server application. 159 The standard approach with a JVM running on top of an Operating System (OS) is 160 shown in Figure 5(a).

161 A JVM without an OS is shown in Figure 5(b). This solution is often called running 162 on the bare metal. The JVM acts as the OS and provides thread scheduling and low163 level access to the hardware. In this case the network stack can be written entirely in 


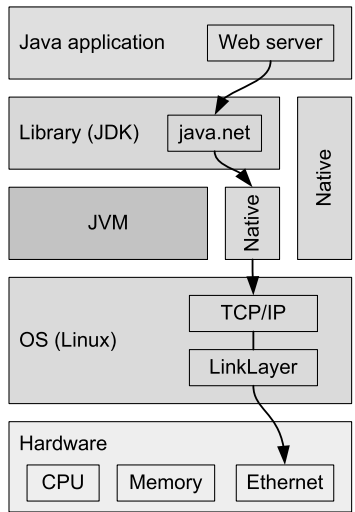

(a)

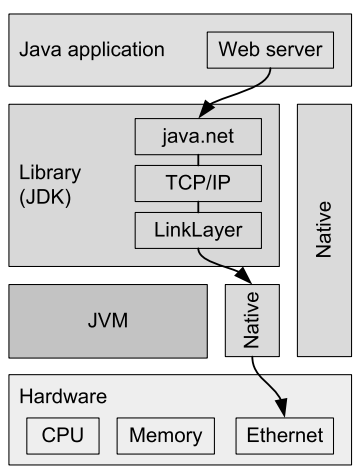

(b)

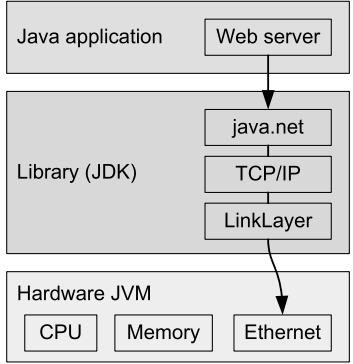

(c)

Fig. 5. Configurations for an embedded JVM: (a) standard layers for Java with an operating system, equivalent to desktop configurations; (b) a JVM on the bare metal; and (c) a JVM as a Java processor.

Java. JNode ${ }^{1}$ is an approach to implement the OS entirely in Java. This solution has become popular even in server applications. ${ }^{2}$

Figure 5(c) shows an embedded solution where the JVM is part of the hardware layer: it is implemented in a Java processor. With this solution the native layer can be completely avoided and all code (application and system code) is written entirely in Java.

Figure 5 shows also the data and control flow from the application down to the hardware. The example consists of a Web server and an Internet connection via Ethernet. In case (a) the application Web server talks with java.net in the Java library. The flow goes down via a native interface to the TCP/IP implementation and the link-layer device driver within the OS (usually written in C). The device driver talks with the Ethernet chip. In (b) the OS layer is omitted: the TCP/IP layer and the link-layer device driver are now part of the Java library. In (c) the JVM is part of the hardware layer, and direct access from the link-layer driver to the Ethernet hardware is mandatory.

With our proposed HAL, as shown in Figure 6, the native interface within the JVM in (a) and (b) disappears. Note how the network stack moves up from the OS layer to the Java library in example (a). All three versions show a pure Java implementation of the whole network stack. The Java code is the same for all three solutions. Version (b) and (c) benefit from hardware objects and interrupt handlers in Java as access to the Ethernet device is required from Java source-code. In Section 5 we show a simple Web server application implemented completely in Java as evaluation of our approach.

\footnotetext{
${ }^{1}$ http: //www . jnode.org/

${ }^{2}$ BEA System offers the JVM LiquidVM that includes basic OS functions and does not need a guest OS.
} 


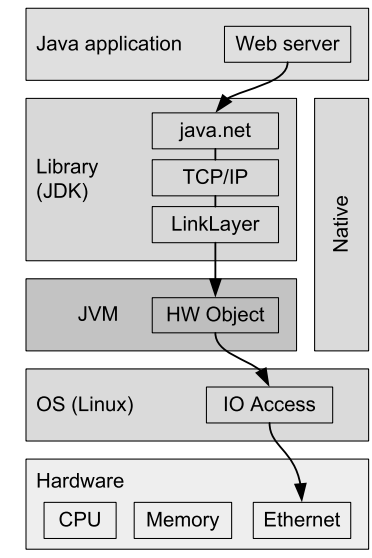

(a)

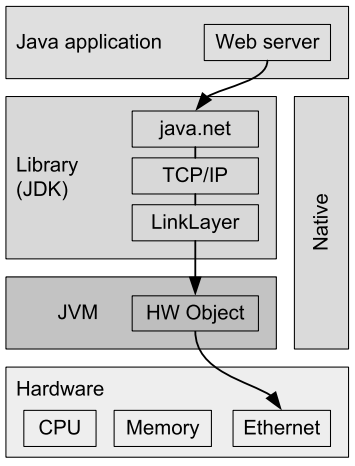

(b)

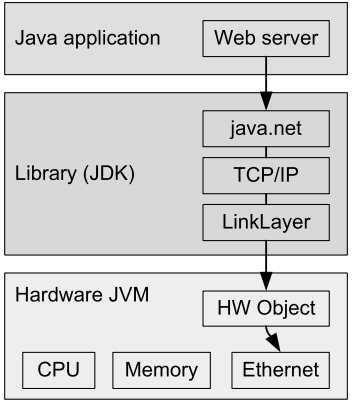

(c)

Fig. 6. Configurations for an embedded JVM with hardware objects and interrupt handlers: (a) standard layers for Java with an operating system, equivalent to desktop configurations; (b) a JVM on the bare metal; and (c) a JVM as a Java processor.

192 Object-oriented. An object representing a device is the most natural integration into 193 an object-oriented language, and a method invocation to a synchronized object is a 194 direct representation of an interrupt.

195 Safe. The safety of Java is not compromised. Hardware objects map object fields to 196 device registers. With a correct class that represents the device, access to it is safe. 197 Hardware objects can be created only by a factory residing in a special package.

198 Generic. The definition of a hardware object and an interrupt handler is independent 199 of the JVM. Therefore, a common standard for different platforms can be defined.

200 Efficient. Device register access is performed by single bytecodes getfield and putfield. 201 We avoid expensive native calls. The handlers are first-level handlers; there is no delay 202 through event queues.

203 The proposed Java HAL would not be useful if it had to be modified for each partic204 ular kind of JVM; thus a second contribution of this article is a number of prototype 205 implementations illustrating the architectures presented in Figure 6: implementa206 tions in Kaffe [Wilkinson 1996] and OVM [Armbruster et al. 2007] represent the 207 architecture with an OS (Figure 6(a)), the implementation in SimpleRTJ [RTJ Com208 puting 2000] represents the bare metal solution (Figure 6(b)), and the implementation 209 in JOP [Schoeberl 2008] represents the Java processor solution (Figure 6(c)).

210 Finally, we must not forget the claim for efficiency, and therefore the article ends 211 with some performance measurements that indicate that the HAL layer is generally 212 as efficient as native calls to $\mathrm{C}$ code external to the JVM.

\section{2. RELATED WORK}

214 Already in the 1970's it was recognized that an operating system might not be the 215 optimal solution for special-purpose applications. Device access was integrated into 216 high-level programming languages like Concurrent Pascal [Hansen 1977; Ravn 1980] 217 and Modula (Modula-2) [Wirth 1977, 1982] along with a number of similar languages, 218 for example, UCSD Pascal. They were meant to eliminate the need for operating sys219 tems and were successfully used in a variety of applications. The programming lan220 guage Ada, which has been dominant in defence and space applications to this day, 
221 may be seen as a continuation of these developments. The advent of inexpensive mi222 croprocessors, from the mid 1980's and on, lead to a regression to assembly and C 223 programming. The hardware platforms were small with limited resources and the de224 velopers were mostly electronic engineers, who viewed them as electronic controllers. 225 Program structure was not considered a major issue in development. Nevertheless, the 226 microcomputer has grown, and is now far more powerful than the minicomputer that 227 it replaced. With powerful processors and an abundance of memory, the ambitions for 228 the functionality of embedded systems grow, and programming becomes a major issue 229 because it may turn out to be the bottleneck in development. Consequently, there is a 230 renewed interest in this line of research.

231 An excellent overview of historical solutions to access hardware devices from and 232 implement interrupt handlers in high-level languages, including $\mathrm{C}$, is presented in 233 Chapter 15 of Burns and Wellings [2001]. The solution to device register access in 234 Modula-1 (Chapter 15.3) is very much like C; however, the constructs are safer because 235 they are encapsulated in modules. Interrupt handlers are represented by threads that 236 block to wait for the interrupt. In Ada (Chapter 15.4) the representation of individual 237 fields in registers can be described precisely by representation classes, while the corre238 sponding structure is bound to a location using the Address attribute. An interrupt is 239 represented in the current version of Ada by a protected procedure, although initially 240 represented (Ada 83) by task entry calls.

241 The main ideas in having device objects are thus found in the earlier safe languages, 242 and our contribution is to align them with a Java model, and in particular, as discussed 243 in Section 4, implementation in a JVM. From the Ada experience we learn that direct 244 handling of interrupts is a desired feature.

\subsection{The Real-Time Specification for Java}

246 The Real-Time Specification for Java (RTSJ) [Bollella et al. 2000] defines a JVM exten247 sion which allows better timeliness control compared to a standard JVM. The core fea248 tures are: fixed priority scheduling, monitors which prevent priority inversion, scoped 249 memory for objects with limited lifetime, immortal memory for objects that are never 250 finalized, and asynchronous events with CPU time consumption control.

251 The RTSJ also defines an API for direct access to physical memory, including hard252 ware registers. Essentially one uses RawMemoryAccess at the level of primitive data 253 types. Although the solution is efficient, this representation of physical memory is not 254 object-oriented, and there are some safety issues: When one raw memory area rep255 resents an address range where several devices are mapped to, there is no protection 256 between them. Yet, a type-safe layer with support for representing individual registers 257 can be implemented on top of the RTSJ API.

258 The RTSJ specification suggests that asynchronous events are used for interrupt 259 handling. Yet, it neither specifies an API for interrupt control nor semantics of the 260 handlers. Any interrupt handling application thus relies on some proprietary API 261 and proprietary event handler semantics. Second-level interrupt handling can be 262 implemented within the RTSJ with an AsyncEvent that is bound to a happening. 263 The happening is a string constant that represents an interrupt, but the meaning 264 is implementation-dependent. An AsyncEventHandler or BoundAsyncEventHandler can 265 be added as handler for the event. Also an AsyncEventHandler can be added via a 266 POSIXSignalHandler to handle POSIX signals. An interrupt handler, written in C, can 267 then use one of the two available POSIX user signals.

268 RTSJ offers facilities very much in line with Modula or Ada for encapsulating 269 memory-mapped device registers. However, we are not aware of any RTSJ implemen270 tation that implements RawMemoryAccess and AsyncEvent with support for low-level 


\subsection{Java Operating Systems}

315 The JX Operating System [Felser et al. 2002] is a microkernel system written mostly 316 in Java. The system consists of components which run in domains, each domain

\footnotetext{
${ }^{3}$ A template can be found at http://practicalembeddedjava.com/tutorials/aJileISR.html.
} 
317 having its own garbage collector, threads, and a scheduler. There is one global preemp318 tive scheduler that schedules the domain schedulers which can be both preemptive 319 and nonpreemptive. Inter-domain communication is only possible through commu320 nication channels exported by services. Low-level access to the physical memory, 321 memory-mapped device registers, and I/O ports are provided by the core ("zero")

domain services, implemented in C. At the Java level ports and memory areas are represented by objects, and registers are methods of these objects. Memory is read and written by access methods of Memory objects. Higher layers of Java interfaces provide type-safe access to the registers; the low-level access is not type safe.

Interrupt handlers in JX are written in Java and are run through portals; they can reside in any domain. Interrupt handlers cannot interrupt the garbage collector (the GC disables interrupts), run with CPU interrupts disabled, must not block, and can only allocate a restricted amount of memory from a reserved per-domain heap. Execution time of interrupt handlers can be monitored: on a deadline violation the handler is aborted and the interrupt source disabled. The first-level handlers can unblock a waiting second-level thread either directly or via setting a state of a AtomicVariable synchronization primitive.

The Java New Operating System Design Effort (JNode ${ }^{4}$ [Lohmeier 2005] is an OS written in Java where the JVM serves as the OS. Drivers are written entirely in Java. Device access is performed via native function calls. A first-level interrupt handler, written in assembler, unblocks a Java interrupt thread. From this thread the device driver-level interrupt handler is invoked with interrupts disabled. Some device drivers implement a synchronized handlelnterrupt(int irq) and use the driver object to signal the upper layer with notifyAll(). During garbage collection all threads are stopped including the interrupt threads.

The Squawk VM [Simon et al. 2006], now available open-source, ${ }^{5}$ is a platform for wireless sensors. Squawk is mostly written in Java and runs without an OS. Device drivers are written in Java and use a form of peek and poke interface to access the device registers. Interrupt handling is supported by a device driver thread that waits for an event from the JVM. The first-level handler, written in assembler, disables the interrupt and notifies the JVM. On a rescheduling point the JVM resumes the device driver thread. It has to reenable the interrupt. The interrupt latency depends on the rescheduling point and on the activity of the garbage collector. For a single device driver thread an average case latency of $0.1 \mathrm{~ms}$ is reported. For a realistic workload with an active garbage collector a worst-case latency of $13 \mathrm{~ms}$ has been observed.

Our proposed constructs should be able to support the Java operating systems. For JX we observe that the concepts are very similar for interrupt handling, and actually for device registers as well. A difference is that we make device objects distinct from memory objects which should give better possibilities for porting to architectures with separate I/O buses. JNode is more traditional and hides first-level interrupt handling and device accesses in the JVM, which may - be less portable than our implementation. The Squawk solution has to have a very small footprint, but on the other hand it can probably rely on having few devices. Device objects would be at least as efficient as the peeks and pokes, and interrupt routines may eliminate the need for multithreading for simple systems, for example, with cyclic executives. Overall, we conclude that our proposed constructs will make implementation of a Java OS more efficient and perhaps more portable.

\footnotetext{
${ }^{4}$ http: //jnode.org/

${ }^{5}$ https ://squawk.dev.java.net/
} 


\subsection{TinyOS and Singularity}

TinyOS [Hill et al. 2000] is an operating system designed for low-power, wireless sensor networks. TinyOS is not a a traditional OS, but provides a framework of components that are linked with the application code. The component-based programming model is supported by nesC [Gay et al. 2003], a dialect of C. TinyOS components provide following abstractions: commands represent requests for a service of a component; events signal the completion of a service; and tasks are functions executed nonpreemptive by the TinyOS scheduler. Events also represent interrupts and preempt tasks. An event handler may post a task for further processing, which is similar to a second-level interrupt handler.

I/O devices are encapsulated in components and the standard distribution of TinyOS includes a rich set of standard I/O devices. A Hardware Presentation Layer (HPL) abstracts the platform-specific access to the hardware (either memory or port mapped). Our proposed HAL is similar to the HPL, but represents the I/O devices as Java objects. A further abstraction into I/O components can be built above our presented Java HAL.

Singularity [Hunt et al. 2005] is a research OS based on a runtime managed language (an extension of $\mathrm{C \# )} \mathrm{to} \mathrm{build} \mathrm{a} \mathrm{software} \mathrm{platform} \mathrm{with} \mathrm{the} \mathrm{main} \mathrm{goal} \mathrm{to} \mathrm{be} \mathrm{de-}$ pendable. A small HAL (loPorts, loDma, lolrq, and loMemory) provides access to PC hardware. C\# style attributes (similar to Java annotations) on fields are used to define the mapping of class fields to I/O ports and memory addresses. The Singularity OS clearly uses device objects and interrupt handlers, thus demonstrating that the ideas presented here transfer to a language like $\mathrm{C \#}$.

\subsection{Summary}

In our analysis of related work we see that our contribution is a selection, adaptation, refinement, and implementation of ideas from earlier languages and platforms for Java. A crucial point, where we have spent much time, is to have a clear interface between the Java layer and the JVM. Here we have used the lessons from the Java OS and the JVM interfaces. Finally, it has been a concern to be consistent with the RTSJ because this standard and adaptations of it are the instruments for developing embedded real-time software in Java.

\section{THE HARDWARE ABSTRACTION LAYER}

In the following section the hardware abstraction layer for Java is defined. Low-level access to devices is performed via hardware objects. Synchronization with a device can be performed with interrupt handlers implemented in Java. Finally, portability of hardware objects, interrupt handlers, and device drivers is supported by a generic configuration mechanism.

\subsection{Device Access}

Hardware objects map object fields to device registers. Therefore, field access with bytecodes putfield and getfield accesses device registers. With a correct class that represents a device, access to it is safe; it is not possible to read or write to an arbitrary memory address. A memory area (e.g., a video frame buffer) represented by an array is protected by Java's array bounds check.

In a C-based system the access to $\mathrm{I} / \mathrm{O}$ devices can either be represented by a $\mathrm{C}$ struct (similar to the class shown in Figure 2) for memory-mapped I/O devices or needs to be accessed by function calls on systems with a separate I/O address space. With the hardware object abstraction in Java the JVM can represent an I/O device as a class independent of the underlying low-level I/O mechanism. Furthermore, the strong 


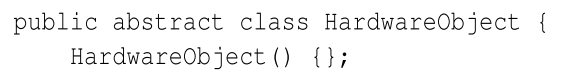

Fig. 7. The marker class for hardware objects.

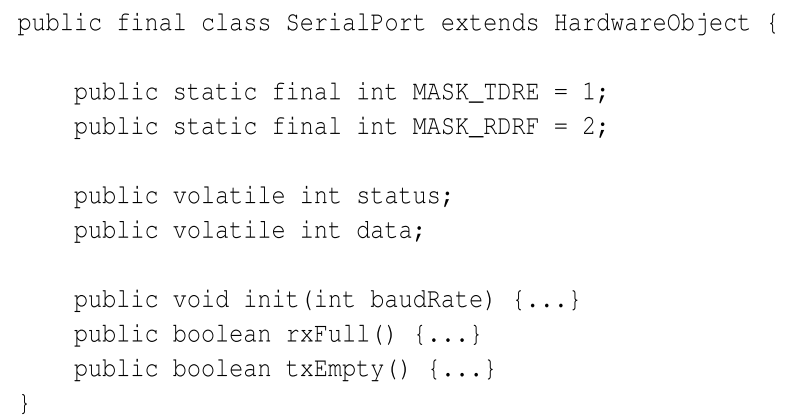

Fig. 8. A serial port class with device methods.

412 typing of Java avoids hard to find programming errors due to wrong pointer casts or 413 wrong pointer arithmetic.

414 All hardware classes have to extend the abstract class HardwareObject (see 415 Figure 7). This empty class serves as type marker. Some implementations use it to 416 distinguish between plain objects and hardware objects for the field access. The 417 package-visible-only constructor disallows creation of hardware objects by the appli418 cation code that resides in a different package. Figure 8 shows a class representing a 419 serial port with a status register and a data register. The status register contains flags 420 for receive register full and transmit register empty; the data register is the receive 421 and transmit buffer. Additionally, we define device-specific constants (bit masks for the 422 status register) in the class for the serial port. All fields represent device registers that 423 can change due to activity of the hardware device. Therefore, they must be declared 424 volatile.

425 In this example we have included some convenience methods to access the hardware 426 object. However, for a clear separation of concerns, the hardware object represents only 427 the device state (the registers). We do not add instance fields to represent additional 428 state, that is, mixing device registers with heap elements. We cannot implement a com429 plete device driver within a hardware object; instead a complete device driver owns a 430 number of private hardware objects along with data structures for buffering, and it 431 defines interrupt handlers and methods for accessing its state from application pro432 cesses. For device-specific operations, such as initialization of the device, methods in 433 hardware objects are useful.

434 Usually each device is represented by exactly one hardware object (see Sec435 tion 3.3.1). However, there might be use cases where this restriction should be re436 laxed. Consider a device where some registers should be accessed by system code only 437 and some other by application code. In JOP there is such a device: a system device 438 that contains a $1 \mathrm{MHz}$ counter, a corresponding timer interrupt, and a watchdog port. 439 The timer interrupt is programmed relative to the counter and used by the real-time 440 scheduler, a JVM internal service. However, access to the counter can be useful for the 441 application code. Access to the watchdog register is required from the application level. 442 The watchdog is used for a sign-of-life from the application. If not triggered every 443 second the complete system is restarted. For this example it is useful to represent one 


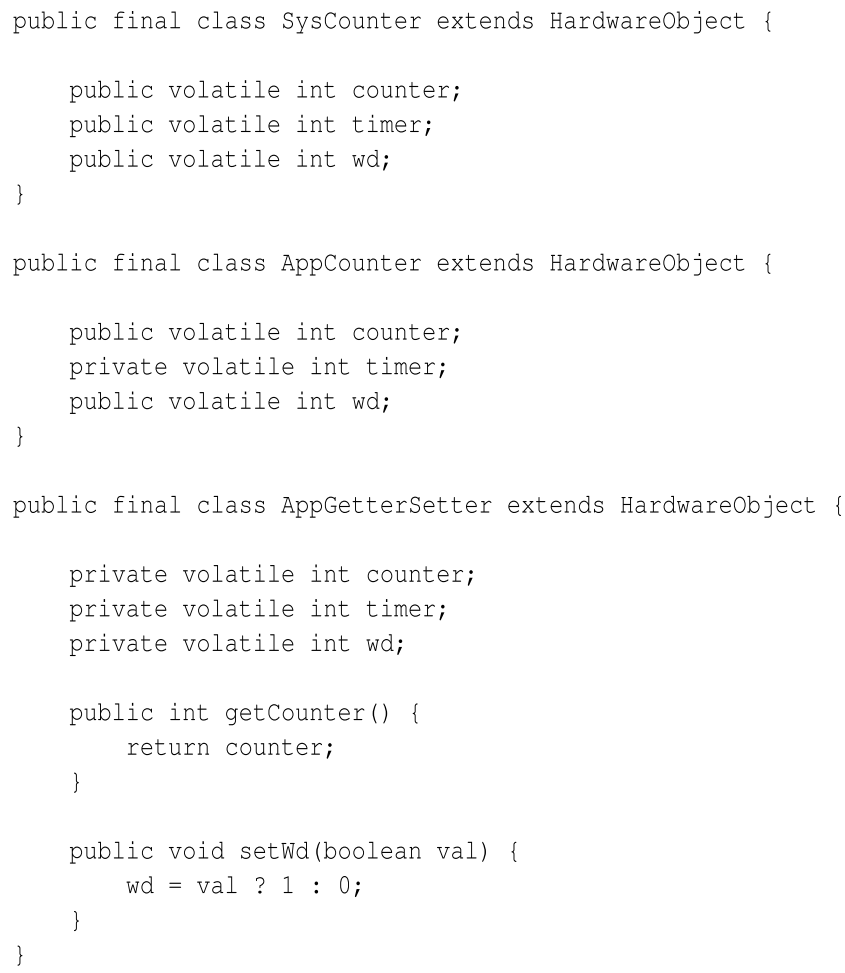

Fig. 9. System and application classes, one with visibility protection and one with setter and getter methods, for a single hardware device

444 hardware device by two different classes: one for system code and one for application 445 code. We can protect system registers by private fields in the hardware object for 446 the application. Figure 9 shows the two class definitions that represent the same 447 hardware device for system and application code respectively. Note how we changed 448 the access to the timer interrupt register to private for the application hardware object. 449 Another option, shown in class AppGetterSetter, is to declare all fields private for 450 the application object and use setter and getter methods. They add an abstraction on 451 top of hardware objects and use the hardware object to implement their functionality. 452 Thus we still do not need to invoke native functions.

453 Use of hardware objects is straightforward. After obtaining a reference to the object 454 all that has to be done (or can be done) is to read from and write to the object fields. 455 Figure 10 shows an example of client code. The example is a Hello World program 456 using low-level access to a serial port via a hardware object. Creation of hardware 457 objects is more complex and described in Section 3.3. Furthermore, it is JVM-specific 458 and Section 4 describes implementations in four different JVMs.

459 For devices that use DMA (e.g., video frame buffer, disk, and network I/O buffers) we 460 map that memory area to Java arrays. Arrays in Java provide access to raw memory 461 in an elegant way: the access is simple and safe due to the array bounds checking 462 done by the JVM. Hardware arrays can be used by the JVM to implement higher-level 463 abstractions from the RTSJ such as RawMemory or scoped memory.

464 Interaction between the Garbage Collector (GC) and hardware objects needs to be 465 crafted into the JVM. We do not want to collect hardware objects. The hardware object 


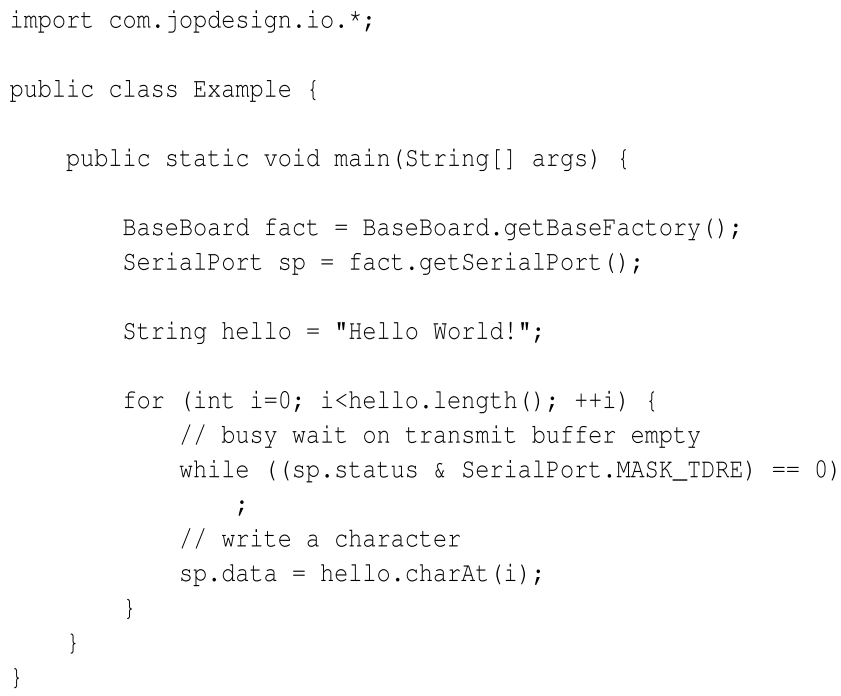

Fig. 10. A "Hello World" example with low-level device access via a hardware object.

Table I. Dispatching Properties of Different ISR Strategies

\begin{tabular}{ccc}
\hline ISR & Context switches & Priorities \\
\hline Handler & 2 & Hardware \\
Event & $3-4$ & Software \\
\hline
\end{tabular}

466 should not be scanned for references. ${ }^{6}$ This is permissible when only primitive types 467 are used in the class definition for hardware objects; the GC scans only reference fields. 468 To avoid collecting hardware objects, we mark the object to be skipped by the GC. The 469 type inheritance from HardwareObject can be used as the marker.

\subsection{Interrupt Handling}

471 An Interrupt Service Routine (ISR) can be integrated with Java in two different ways: 472 as a first-level handler or a second-level event handler.

473 ISR handler. The interrupt is a method call initiated by the device.

474 Usually this abstraction is supported in hardware by the processor and called a 475 first-level handler.

476 ISR event. The interrupt is represented by an asynchronous notification directed to 477 a thread that is unblocked from a wait-state. This is also called deferred interrupt 478 handling.

479 An overview of the dispatching properties of the two approaches is given in Table I. 480 The ISR handler approach needs only two context switches and the priority is set 481 by the hardware. With the ISR event approach, handlers are scheduled at software 482 priorities. The initial first-level handler, running at hardware priority, fires the event

\footnotetext{
${ }^{6}$ If a hardware coprocessor, represented by a hardware object, itself manipulates an object on the heap and holds the only reference to that object it has to be scanned by the GC.
} 
483 for the event handler. Also the first-level handler will notify the scheduler. In the best 484 case three context switches are necessary: one to the first-level handler, one to the ISR 485 event handler, and one back to the interrupted thread. If the ISR handler has a lower 486 priority than the interrupted thread, an additional context switch from the first-level 487 handler back to the interrupted thread is necessary.

488 Another possibility is to represent an interrupt as a thread that is released by the 489 interrupt. Direct support by the hardware (e.g., the interrupt service thread in Ko490 modo [Kreuzinger et al. 2003]) gives fast interrupt response times. However, standard 491 processors support only the handler model directly.

492 Direct handling of interrupts in Java requires the JVM to be prepared to be inter493 rupted. In an interpreting JVM an initial handler will reenter the JVM to execute the 494 Java handler. A compiling JVM or a Java processor can directly invoke a Java method 495 as response to the interrupt. A compiled Java method can be registered directly in the 496 ISR dispatch table.

497 If an internal scheduler is used (also called green threads) the JVM will need some 498 refactoring in order to support asynchronous method invocation. Usually JVMs control 499 the rescheduling at the JVM level to provide a lightweight protection of JVM internal 500 data structures. These preemption points are called pollchecks or yield points; also 501 some or all can be GC preemption points. In fact the preemption points resemble 502 cooperative scheduling at the JVM level and use priority for synchronization. This 503 approach works only for uniprocessor systems; for multiprocessors explicit synchro504 nization has to be introduced.

505 In both cases there might be critical sections in the JVM where reentry cannot 506 be allowed. To solve this problem the JVM must disable interrupts around critical 507 nonreenterable sections. The more fine-grained this disabling of interrupts can be 508 done, the more responsive to interrupts the system will be.

509 One could opt for second-level handlers only. An interrupt fires and releases an 510 associated schedulable object (handler). Once released, the handler will be sched511 uled by the JVM scheduler according to the release parameters. This is the RTSJ 512 approach. The advantage is that interrupt handling is done in the context of a normal 513 Java thread and scheduled as any other thread running on the system. The drawback 514 is that there will be a delay from the occurrence of the interrupt until the thread gets 515 scheduled. Additionally, the meaning of interrupt priorities, levels, and masks used by 516 the hardware may not map directly to scheduling parameters supported by the JVM 517 scheduler.

518 In the following we focus on the ISR handler approach, because it allows program519 ming the other paradigms within Java.

520 3.2.1 Hardware Properties. We assume interrupt hardware as it is found in most com521 puter architectures: interrupts have a fixed priority associated with them; they are 522 set with a solder iron. Furthermore, interrupts can be globally disabled. In most 523 systems the first-level handler is called with interrupts globally disabled. To allow 524 nested interrupts (being able to interrupt the handler by a higher-priority interrupt 525 as in preemptive scheduling) the handler has to enable interrupts again. However, to 526 avoid priority inversion between handlers only interrupts with a higher priority will 527 be enabled, either by setting the interrupt level or setting the interrupt mask. Soft528 ware threads are scheduled by a timer interrupt and usually have a lower priority 529 than interrupt handlers (the timer interrupt has the lowest priority of all interrupts). 530 Therefore, an interrupt handler is never preempted by a software thread.

531 Mutual exclusion between an interrupt handler and a software thread is ensured by 532 disabling interrupts: either all interrupts or selectively. Again, to avoid priority inver533 sion, only interrupts of a higher priority than the interrupt that shares the data with 


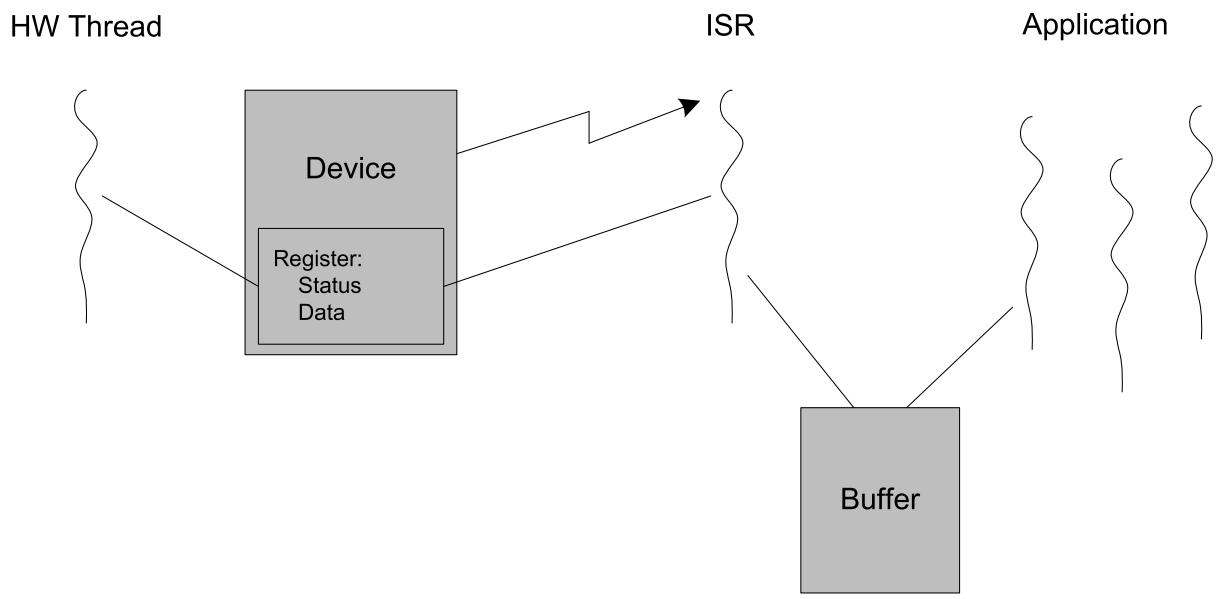

Fig. 11. Threads and shared data.

534 the software thread can be enabled. This mechanism is in effect the priority ceiling emulation protocol [Sha et al. 1990], sometimes called immediate ceiling protocol. It 536 has the virtue that it eliminates the need for explicit locks (or Java monitors) on shared 537 objects. Note that mutual exclusion with interrupt disabling works only in a unipro538 cessor setting. A simple solution for multiprocessors is to run the interrupt handler 539 and associated software threads on the same processor core. A more involved scheme 540 would be to use spin-locks between the processors.

541 When a device asserts an interrupt request line, the interrupt controller notifies the 542 processor. The processor stops execution of the current thread. A partial thread con543 text (program counter and processor status register) is saved. Then the ISR is looked 544 up in the interrupt vector table and a jump is performed to the first instruction of the 545 ISR. The handler usually saves additional thread context (e.g., the register file). It is 546 also possible to switch to a new stack area. This is important for embedded systems 547 where the stack sizes for all threads need to be determined at link time.

3.2.2 Synchronization. Java supports synchronization between Java threads with the synchronized keyword, either as a means of synchronizing access to a block of statements or to an entire method. In the general case this existing synchronization support is not sufficient to synchronize between interrupt handlers and threads.

Figure 11 shows the interacting active processes and the shared data in a scenario 553 involving the handling of an interrupt. Conceptually three threads interact: (1) a hard554 ware device thread representing the device activity, (2) the ISR, and (3) the application or device driver thread. These three share two types of data.

556 Device data. The hardware thread and ISR share access to the device registers of the 557 device signaling the interrupt.

558 Application data. The ISR and application or device driver share access to, for exam559 ple, a buffer conveying information about the interrupt to the application.

560 Regardless of which interrupt handling approach is used in Java, synchronization 561 between the ISR and the device registers must be handled in an ad hoc way. In gen562 eral there is no guarantee that the device has not changed the data in its registers; 563 but if the ISR can be run to completion within the minimum inter-arrival time of the 564 interrupt the content of the device registers can be trusted. 
For synchronization between the ISR and the application (or device driver) the following mechanisms are available. When the ISR handler runs as a software thread, standard synchronization with object monitors can be used. When using the ISR handler approach, the handler is no longer scheduled by the normal Java scheduler, but by the hardware. While the handler is running, all other executable elements are suspended, including the scheduler. This means that the ISR cannot be suspended, must not block, or must not block via a language-level synchronization mechanism; the ISR must run to completion in order not to freeze the system. This means that when the handler runs, it is guaranteed that the application will not get scheduled. It follows that the handler can access data shared with the application without synchronizing with the application. As the access to the shared data by the interrupt handler is not explicitly protected by a synchronized method or block, the shared data needs to be declared volatile.

On the other hand the application must synchronize with the ISR because the ISR may be dispatched at any point. To ensure mutual exclusion we redefine the semantics of the monitor associated with an InterruptHandler object: acquisition of the monitor disables all interrupts of the same and lower priority; release of the monitor enables the interrupts again. This procedure ensures that the software thread cannot be interrupted by the interrupt handler when accessing shared data.

3.2.3 Using the Interrupt Handler. Figure 12 shows an example of an interrupt handler for the serial port receiver interrupt. The method handle() is the interrupt handler method and needs no synchronization as it cannot be interrupted by a software thread. However, the shared data needs to be declared volatile as it is changed by the device driver thread. Method read() is invoked by the device driver thread and the shared data is protected by the InterruptHandler monitor. The serial port interrupt handler uses the hardware object SerialPort to access the device.

3.2.4 Garbage Collection. When using the ISR handler approach it is not feasible to let interrupt handlers be paused during a lengthy stop-the-world collection. Using this GC strategy the entire heap is collected at once and it is not interleaved with execution. The collector can safely assume that data required to perform the collection will not change during the collection, and an interrupt handler shall not change data used by the GC to complete the collection. In the general case, this means that the interrupt handler is not allowed to create new objects, or change the graph of live objects.

With an incremental GC the heap is collected in small incremental steps. Write barriers in the mutator threads and nonpreemption sections in the GC thread synchronize the view of the object graph between the mutator threads and the GC thread. With incremental collection it is possible to allow object allocation and changing references inside an interrupt handler (as it is allowed in any normal thread). With a real-time GC the maximum blocking time due to GC synchronization with the mutator threads must be known.

Interruption of the GC during an object move can result in access to a stale copy of the object inside the handler. A solution to this problem is to allow for pinning of objects reachable by the handler (similar to immortal memory in the RTSJ). Concurrent collectors have to solve this issue for threads anyway. The simplest approach is to disable interrupt handling during the object copy. As this operation can be quite long for large arrays, several approaches to split the array into smaller chunks have been proposed [Bacon et al. 2003; Siebert 2002]. A Java processor may support incremental array copying with redirection of the access to the correct part of the array [Schoeberl and Puffitsch 2008]. Another solution is to abort the object copy when writing to the 


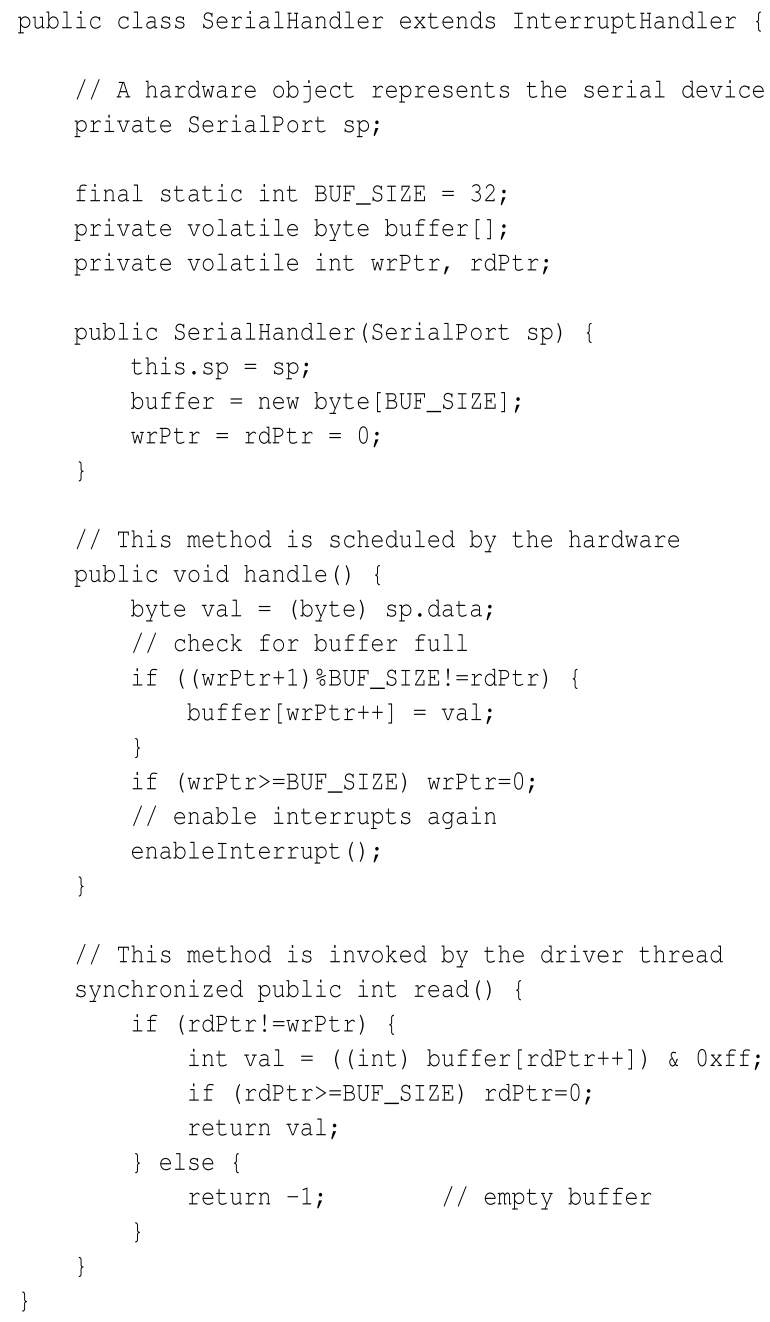

Fig. 12. An interrupt handler for a serial port receive interrupt.

615 object. It is also possible to use replication; during an incremental copy operation, 616 writes are performed on both from-space and to-space object replicas, while reads are 617 performed on the from-space replica.

\section{3.3 Generic Configuration}

619 An important issue for a HAL is a safe abstraction of device configurations. A def620 inition of factories to create hardware and interrupt objects should be provided by 621 board vendors. This configuration is isolated with the help of Java packages; only the 622 objects and the factory methods are visible. The configuration abstraction is indepen623 dent of the JVM. A device or interrupt can be represented by an identical hardware 624 or interrupt object for different JVMs. Therefore, device drivers written in Java are 625 JVM-independent.

626 3.3.1 Hardware Object Creation. The idea to represent each device by a single object or 627 array is straightforward, the remaining question is: How are those objects created? 


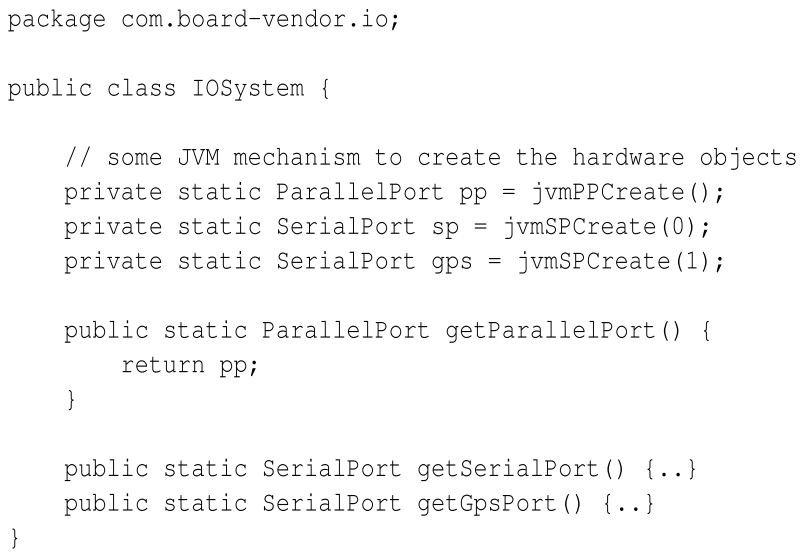

Fig. 13. A factory with static methods for Singleton hardware objects.

628 An object that represents a device is a typical Singleton [Gamma et al. 1994]. Only 629 a single object should map to one instance of a device. Therefore, hardware objects 630 cannot be instantiated by a simple new: (1) they have to be mapped by some JVM 631 mechanism to the device registers and (2) each device instance is represented by a 632 single object.

633 Each device object is created by its own factory method. The collection of these 634 methods is the board configuration, which itself is also a Singleton (the application 635 runs on a single board). The Singleton property of the configuration is enforced by 636 a class that contains only static methods. Figure 13 shows an example for such a 637 class. The class IOSystem represents a system with three devices: a parallel port, 638 as discussed before to interact with the environment, and two serial ports: one for 639 program download and one which is an interface to a GPS receiver.

640 This approach is simple, but not very flexible. Consider a vendor who provides 641 boards in slightly different configurations (e.g., with different number of serial ports). 642 With the preceding approach each board requires a different (or additional) IOSystem 643 class that lists all devices. A more elegant solution is proposed in the next section.

644 3.3.2 Board Configurations. Replacing the static factory methods by instance methods 645 avoids code duplication; inheritance then gives configurations. With a factory object 646 we represent the common subset of I/O devices by a base class and the variants as 647 subclasses.

648 However, the factory object itself must still be a Singleton. Therefore the board649 specific factory object is created at class initialization and is retrieved by a static 650 method. Figure 14 shows an example of a base factory and a derived factory. Note 651 how getBaseFactory() is used to get a single instance of the factory. We have applied 652 the idea of a factory two times: the first factory generates an object that represents 653 the board configuration. That object is itself a factory that generates the objects that 654 interface to the hardware device.

655 The shown example base factory represents the minimum configuration with a sin656 gle serial port for communication (mapped to System.in and System.out) represented 657 by a SerialPort. The derived configuration ExtendedBoard contains an additional serial 658 port for a GPS receiver and a parallel port for external control.

659 Furthermore, we show in Figure 14 a different way to incorporate the JVM mech660 anism in the factory: we define well-known constants (the memory addresses of the 


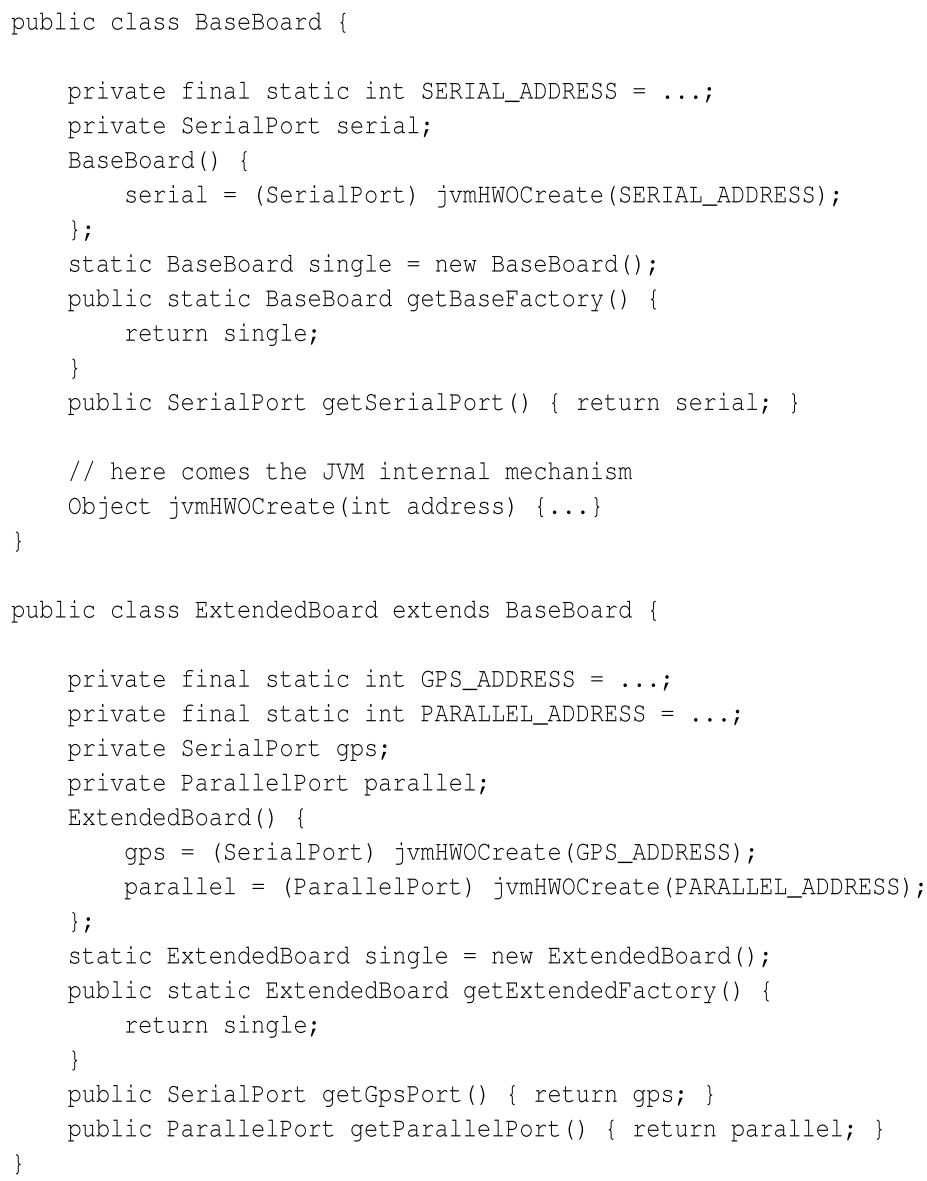

Fig. 14. A base class of a hardware object factory and a factory subclass.

661 devices) in the factory and let the native function jvmHWOCreate() return the correct 662 device type.

663 Figure 15 gives a summary example of hardware object classes and the correspond664 ing factory classes as an UML class diagram. The figure shows that all device classes 665 subclass the abstract class HardwareObject. Figure 15 represents the simple abstrac666 tion as it is seen by the user of hardware objects.

667 3.3.3 Interrupt Handler Registration. We provide a base interrupt handling API that can 668 be used both for non-RTSJ and RTSJ interrupt handling. The base class that is ex669 tended by an interrupt handler is shown in Figure 16. The handle() method contains 670 the device server code. Interrupt control operations that have to be invoked before 671 serving the device (i.e., interrupt masking and acknowledging) and after serving the 672 device (i.e., interrupt reenabling) are hidden in the run() method of the base Inter673 ruptHandler, which is invoked when the interrupt occurs.

674 The base implementation of InterruptHandler also provides methods for enabling and 675 disabling a particular interrupt or all local CPU interrupts and a special monitor im676 plementation for synchronization between an interrupt handler thread and an applica677 tion thread. Moreover, it provides methods for non-RTSJ registering and deregistering 678 the handler with the hardware interrupt source. 


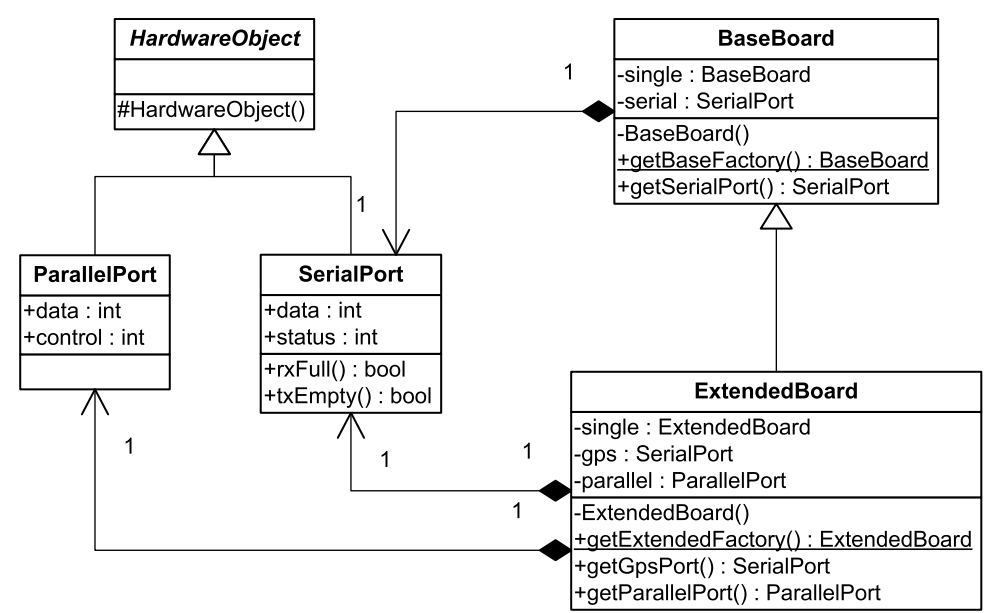

Fig. 15. Device object classes and board factory classes.

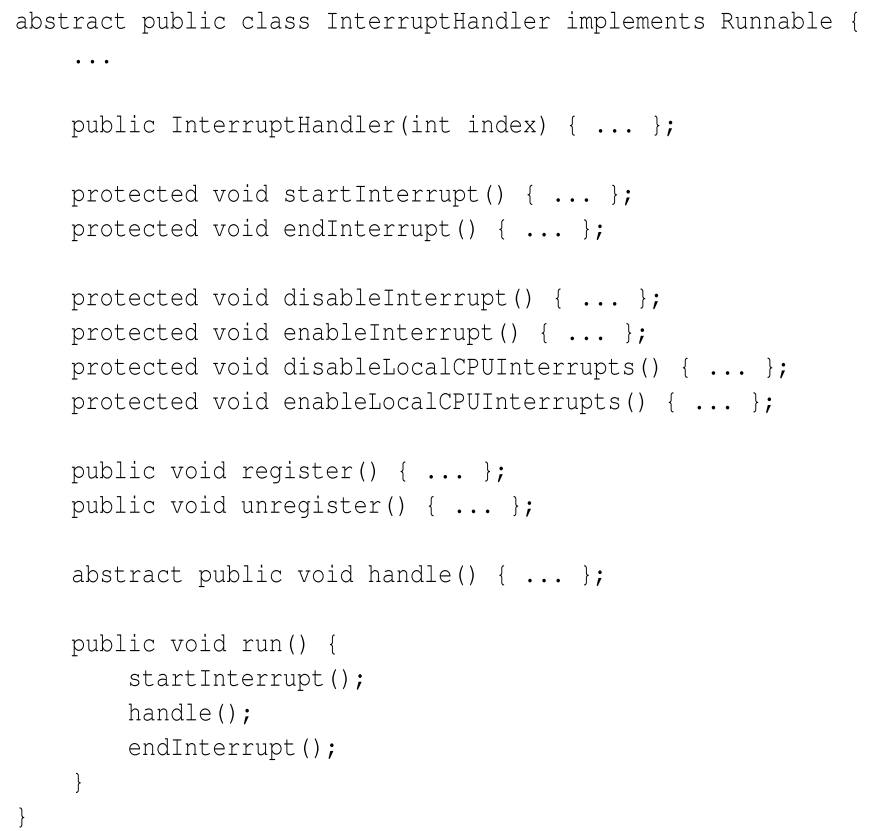

Fig. 16. Base class for the interrupt handlers.

679 Registration of a RTSJ interrupt handler requires more steps (see Figure 17). The 680 InterruptHandler instance serves as the RTSJ logic for a (bound) asynchronous event 681 handler, which is added as handler to an asynchronous event which then is bound to 682 the interrupt source.

\section{3.4 Perspective}

684 An interesting topic is to define a common standard for hardware objects and interrupt 685 handlers for different platforms. If different device types (hardware chips) that do not 686 share a common register layout are used for a similar function, the hardware objects 


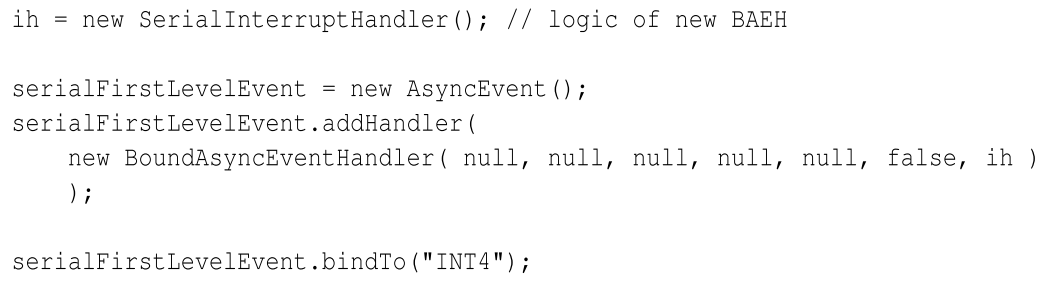

Fig. 17. Creation and registration of a RTSJ interrupt handler.

Table II. Embedded Java Architectures

\begin{tabular}{ccc}
\hline & Direct (no OS) & Indirect (OS) \\
\hline Interpreted & SimpleRTJ & Kaffe VM \\
Native & JOP & OVM \\
\hline
\end{tabular}

687 will be different. However, if the structure of the devices is similar, as is the case 688 for the serial port on the three different platforms used for the implementation (see 689 Section 4), the driver code that uses the hardware object is identical.

690 If the same chip (e.g., the 8250 type and compatible 16x50 devices found in all PCs 691 for the serial port) is used in different platforms, the hardware object and the device 692 driver, which also implements the interrupt handler, can be shared. The hardware ob693 ject, the interrupt handler, and the visible API of the factory classes are independent of 694 the JVM and the OS. Only the implementation of the factory methods is JVM-specific. 695 Therefore, the JVM-independent HAL can be used to start the development of drivers 696 for a Java OS on any JVM that supports the proposed HAL.

\section{3.5 Summary}

698 Hardware objects are easy to use for a programmer, and the corresponding definitions 699 are comparatively easy to define for a hardware designer or manufacturer. For a stan700 dardized HAL architecture we proposed factory patterns. As shown, interrupt han701 dlers are easy to use for a programmer who knows the underlying hardware paradigm, 702 and the definitions are comparatively easy to develop for a hardware designer or man703 ufacturer, for instance using the patterns outlined in this section. Hardware objects 704 and interrupt handler infrastructure have a few subtle implementation points which 705 are discussed in the next section.

\section{4. IMPLEMENTATION}

707 We have implemented the core concepts on four different $\mathrm{JVMs}^{7}$ to validate the pro708 posed Java HAL. Table II classifies the four execution environments according to two 709 important properties: (1) whether they run on bare metal or on top of an OS and (2) 710 whether Java code is interpreted or executed natively. Thereby we cover the whole 711 implementation spectrum with our four implementations. Even though the suggested 712 Java HAL is intended for systems running on bare metal, we include systems run713 ning on top of an OS because most existing JVMs still require an OS, and in order for 714 them to migrate incrementally to run directly on the hardware they can benefit from 715 supporting a Java HAL.

716 In the direct implementation a JVM without an OS is extended with I/O function717 ality. The indirect implementation represents an abstraction mismatch; we actually

\footnotetext{
${ }^{7} \mathrm{On}$ JOP the implementation of the Java HAL is already in use in production code.
} 


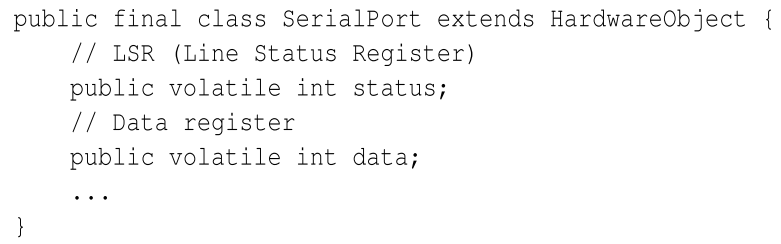

Fig. 18. A simple hardware object.

718 remap the concepts. Related to Figure 6 in the Introduction, OVM and Kaffe represent configuration (a), SimpleRTJ configuration (b), and JOP configuration (c).

720 The SimpleRTJ JVM [RTJ Computing 2000] is a small, interpreting JVM that does 721 not require an OS. JOP [Schoeberl 2005, 2008] is a Java processor executing Java bytecodes directly in hardware. Kaffe JVM [Wilkinson 1996] is a complete, full-featured JVM supporting both interpretation and JIT compilation; in our experiments with Kaffe we have used interpretative execution only. The OVM JVM [Armbruster et al. 2007] is an execution environment for Java that supports compilation of Java bytecodes into the $\mathrm{C}$ language, and via a $\mathrm{C}$ compiler into native machine instructions for the target hardware. Hardware objects have also been implemented in the research , CACAO [Krall and Grafl 1997; Schoeberl et al. 2008].

In the following we provide the different implementation approaches that are necessary for the very different JVMs. Implementing hardware objects was straightforward for most JVMs; it took about one day to implement them in JOP. In Kaffe, after familing us with the structure of the JVM, it took about half a day of pair programming.

Interrupt handling in Java is straightforward in a JVM not running on top of an OS (JOP and SimpleRTJ). Kaffe and OVM both run under vanilla Linux or the realtime version Xenomai Linux [Xenomai Developers 2008]. Both versions use a distinct user/kernel mode and it is not possible to register a user-level method as interrupt ndler. Therefore, we used threads at different levels to simulate the Java handler approach. The result is that the actual Java handler is the third- or even fourth-level handler. This solution introduces quite a lot of overheads due to the many context Java; the goal is a real-time JVM that runs on the bare hardware.

In this section we provide more implementation details than usual to help other JVM developers to add a HAL to their JVM. The techniques used for the JVMs can probably not be used directly. However, the solutions (or sometimes work-arounds) presented here should give enough insight to guide other JVM developers.

746

747

\subsection{SimpleRTJ}

The SimpleRTJ JVM is a small, simple, and portable JVM. We have ported it to run on the bare metal of a small 16-bit microcontroller. We have successfully implemented the support for hardware objects in the SimpleRTJ JVM. For interrupt handling we use the ISR handler approach described in Section 3.2. Adding support for hardware objects was straightforward, but adding support for interrupt handling required more work.

4.1.1 Hardware Objects. Given an instance of a hardware object as shown in Figure 18 one must calculate the base address of the I/O port range, the offset to the actual I/O port, and the width of the port at runtime. We have chosen to store the base address of the I/O port range in a field in the common superclass for all hardware objects (HardwareObject). The hardware object factory passes the platform- and device-specific 


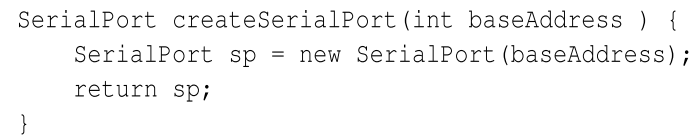

Fig. 19. Creating a simple hardware object.

$784 \mathrm{JOP}$ is a Java processor intended for hard real-time systems [Schoeberl 2005, 2008] 785 All architectural features have been carefully designed to be time-predictable with 786 minimal impact on average case performance. We have implemented the proposed 787 HAL in the JVM for JOP. No changes inside the JVM (the microcode in JOP) were 788 necessary. Only the creation of the hardware objects needs a JOP-specific factory.

789 4.2.1 Hardware Objects. In JOP, objects and arrays are referenced through an indirec790 tion called handle. This indirection is a lightweight read barrier for the compacting 791 real-time GC [Schoeberl 2006; Schoeberl and Vitek 2007]. All handles for objects in the 792 heap are located in a distinct memory region, the handle area. Besides the indirection 793 to the real object the handle contains auxiliary data, such as a reference to the class 794 information, the array length, and GC-related data. Figure 20 shows an example with 795 a small object that contains two fields and an integer array of length 4 . The object and 796 the array on the heap just contain the data and no additional hidden fields. This object 797 layout greatly simplifies our object to device mapping. We just need a handle where 798 the indirection points to the memory-mapped device registers instead of into the heap. 799 This configuration is shown in the upper part of Figure 20. Note that we do not need 


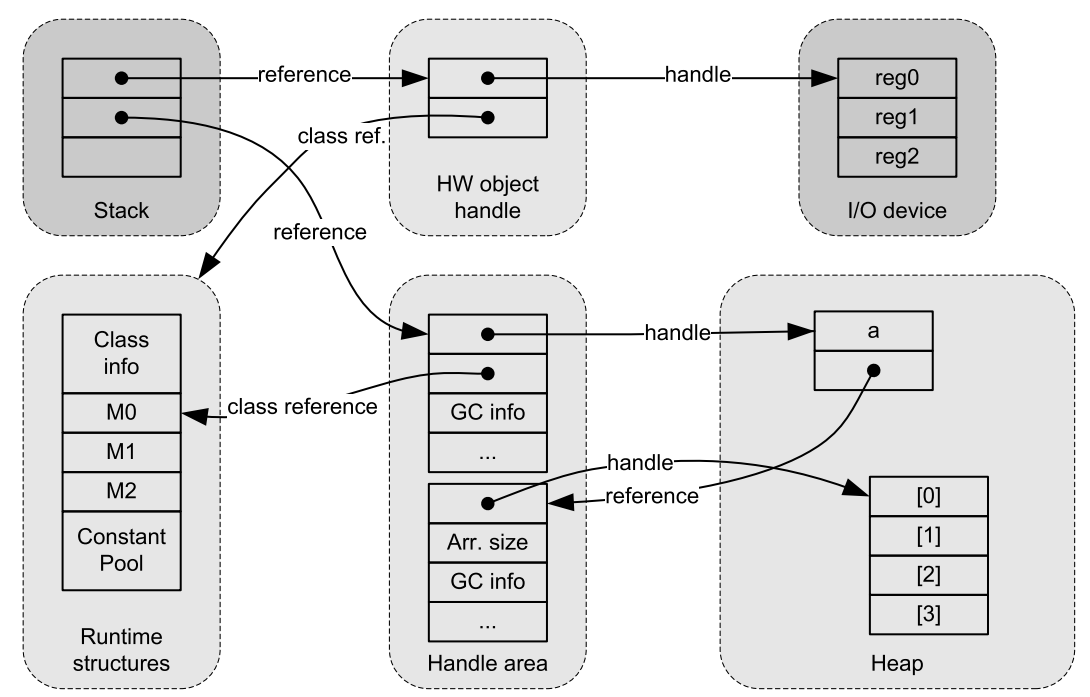

Fig. 20. Memory layout of the JOP JVM.

800 the GC information for the hardware object handles. The factory, which creates the 801 hardware objects, implements this indirection.

802 As described in Section 3.3.1 we do not allow applications to create hardware objects; 803 the constructor is private (or package visible). Figure 21 shows part of the hardware 804 object factory that creates the hardware object SerialPort. Two static fields (SP_PTR 805 and SP_MTAB) are used to store the handle to the serial port object. The first field is 806 initialized with the base address of the I/O device; the second field contains a pointer 807 to the class information. ${ }^{8}$ The address of the static field SP_PTR is returned as the 808 reference to the serial port object.

809 The class reference for the hardware object is obtained by creating a normal instance 810 of SerialPort with new on the heap and copying the pointer to the class information. To 811 avoid using native methods in the factory class we delegate JVM internal work to a 812 helper class in the JVM system package as shown in Figure 21. That helper method 813 returns the address of the static field SP_PTR as reference to the hardware object. 814 All methods in class Native, a JOP system class, are native ${ }^{9}$ methods for low-level 815 functions, the code we want to avoid in application code. Method tolnt(Object o) defeats 816 Java's type safety and returns a reference as an int. Method toObject(int addr) is the 817 inverse function to map an address to a Java reference. Low-level memory access 818 methods are used to manipulate the JVM data structures.

819 To disallow the creation with new in normal application code, the visibility is set 820 to package. However, the package visibility of the hardware object constructor is a 821 minor issue. To access private static fields of an arbitrary class from the system class 822 we had to change the runtime class information: we added a pointer to the first static 823 primitive field of that class. As addresses of static fields get resolved at class linking, 824 no such reference was needed so far.

\footnotetext{
${ }^{8}$ In JOP's JVM the class reference is a pointer to the method table to speed-up the invoke instruction. Therefore, the name is XX_MTAB.

${ }^{9}$ There are no real native functions in JOP; bytecode is the native instruction set. The very few native methods in class Native are replaced by special, unused bytecodes during class linking.
} 


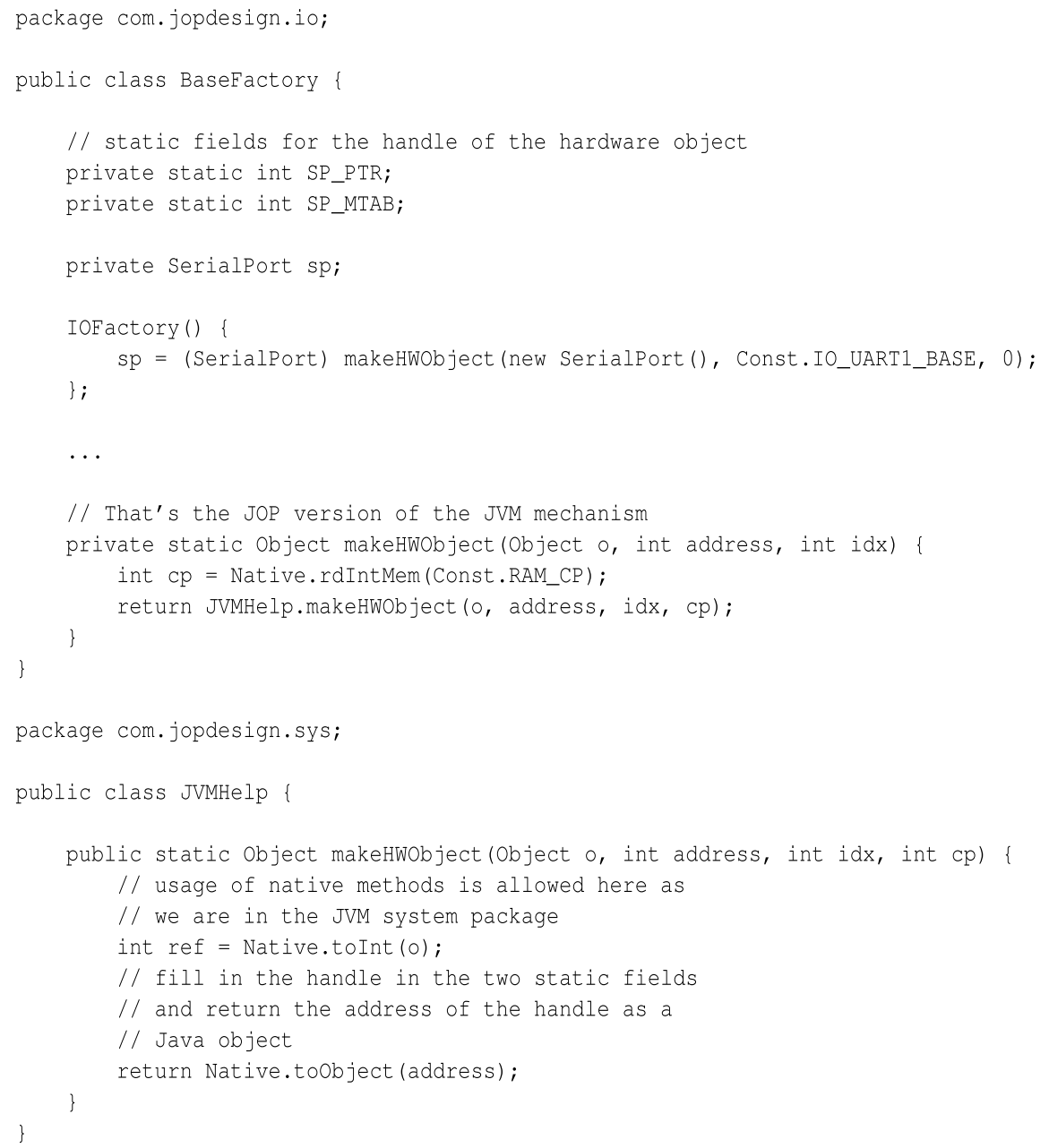

Fig. 21. Part of a factory and the helper method for the hardware object creation in the factory.

4.2.2 Interrupt Handler. The original JOP [Schoeberl 2005, 2008] was a very puristic 826 hard real-time processor. There existed only one interrupt: the programmable timer 827 interrupt as time is the primary source for hard real-time events. All I/O requests were 828 handled by periodic threads that polled for pending input data or free output buffers. 829 During the course of this research we have added an interrupt controller to JOP and 830 the necessary software layers.

831 Interrupts and exact exceptions are considered the hard part in the implementa832 tion of a processor pipeline [Hennessy and Patterson 2002]. The pipeline has to be 833 drained and the complete processor state saved. In JOP there is a translation stage 834 between Java bytecodes and the JOP internal microcode [Schoeberl 2008]. On a pend835 ing interrupt (or exception generated by the hardware) we use this translation stage 836 to insert a special bytecode in the instruction stream. This approach keeps the inter837 rupt completely transparent to the core pipeline. The special bytecode that is unused 838 by the JVM specification [Lindholm and Yellin 1999] is handled in JOP as any other 839 bytecode: execute microcode, invoke a special method from a helper class, or execute 


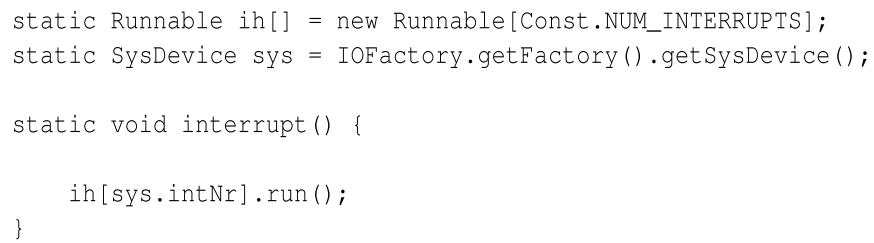

Fig. 22. Interrupt dispatch with the static interrupt() method in the JVM helper class.

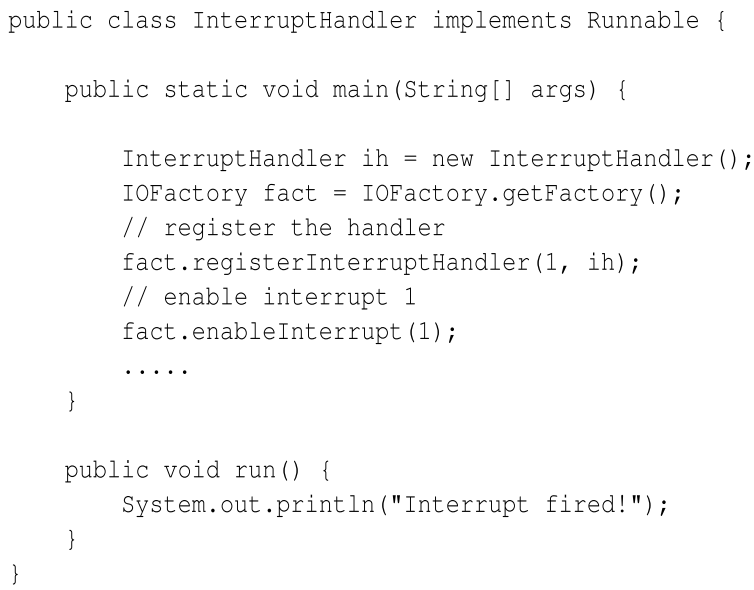

Fig. 23. An example Java interrupt handler as Runnable.

840 Java bytecode from JVM.java. In our implementation we invoke the special method 841 interrupt() from a JVM helper class.

842 The implemented Interrupt Controller (IC) is priority-based. The number of inter843 rupt sources can be configured. Each interrupt can be triggered in software by a IC 844 register write as well. There is one global interrupt enable and each interrupt line can 845 be enabled or disabled locally. The interrupt is forwarded to the bytecode/microcode 846 translation stage with the interrupt number. When accepted by this stage, the inter847 rupt is acknowledged and the global enable flag cleared. This feature avoids immediate 848 handling of an arriving higher-priority interrupt during the first part of the handler. 849 The interrupts have to be enabled again by the handler at a convenient time. All inter850 rupts are mapped to the same special bytecode. Therefore, we perform the dispatch of 851 the correct handler in Java. On an interrupt the static method interrupt() from a system 852 internal class gets invoked. The method reads the interrupt number and performs the 853 dispatch to the registered Runnable as illustrated in Figure 22. Note how a hardware 854 object of type SysDevice is used to read the interrupt number.

855 The timer interrupt, used for the real-time scheduler, is located at index 0 . The 856 scheduler is just a plain interrupt handler that gets registered at mission start at index 8570 . At system startup, the table of Runnables is initialized with dummy handlers. The 858 application code provides the handler via a class that implements Runnable and regis859 ters that class for an interrupt number. We reuse the factory presented in Section 3.3.1. 860 Figure 23 shows a simple example of an interrupt handler implemented in Java.

861 For interrupts that should be handled by an event handler under the control of the 862 scheduler, the following steps need to be performed on JOP.

863 (1) Create a SwEvent with the correct priority that performs the second-level interrupt 864 handler work. 
(2) Create a short first-level interrupt handler as Runnable that invokes fire() of the corresponding software event handler.

(3) Register the first-level interrupt handler as shown in Figure 23 and start the realtime scheduler.

In Section 5 we evaluate the different latencies of first- and second-level interrupt handlers on JOP.

\subsection{Kaffe}

Kaffe is an open-source ${ }^{10}$ implementation of the JVM which makes it possible to add support for hardware objects and interrupt handlers. Kaffe requires a fully fledged OS such as Linux to compile and run. Although ports of Kaffe exist on uCLinux we have not been able to find a bare metal version of Kaffe. Thus even though we managed to add support of hardware objects and interrupt handling to Kaffe, it still cannot be used without an OS.

4.3.1 Hardware Objects. Hardware objects have been implemented in the same manner as in the SimpleRTJ, described in Section 4.1.

4.3.2 Interrupt Handler. Since Kaffe runs under Linux we cannot directly support the ISR handler approach. Instead we used the ISR event approach in which a thread blocks waiting for the interrupt to occur. It turned out that the main implementation effort was spent in the signaling of an interrupt occurrence from the kernel space to the user space.

We wrote a special Linux kernel module in the form of a character device. Through proper invocations of ioctl() it is possible to let the module install a handler for an interrupt (e.g., the serial interrupt, normally on IRQ 7). Then the Kaffe VM can make a blocking call to read() on the proper device. Finally the installed kernel handler will release the user space application from the blocked call when an interrupt occurs.

Using this strategy we have performed nontrivial experiments implementing a full interrupt handler for the serial interrupt in Java. Still, the elaborate setup requiring a special-purpose kernel device is far from our ultimate goal of running a JVM on the bare metal. Nevertheless the experiment has given valuable experience with interrupt handlers and hardware objects at the Java language level.

\subsection{OVM}

OVM [Armbruster et al. 2007] is a research JVM allowing many configurations; it is primarily targeted at implementing a large subset of RTSJ while maintaining reasonable performance. OVM uses ahead of time compilation via the C language: it translates both application and VM bytecodes to $\mathrm{C}$, including all classes that might be later loaded dynamically at runtime. The $\mathrm{C}$ code is then compiled by GCC.

4.4.1 Hardware Objects. To compile Java bytecode into a C program, the OVM's Javato- $\mathrm{C}$ compiler internally converts the bytecode into an Intermediate Representation (IR) which is similar to the bytecode, but includes more codes. Transformations at the IR level are both optimizations and operations necessary for correct execution, such as insertion of null-pointer checks. The produced IR is then translated into $\mathrm{C}$, allowing the $\mathrm{C}$ compiler to perform additional optimizations. Transformations at the IR level, which is similar to the bytecode, are also typical in other JVM implementations, such as Sun's HotSpot.

${ }^{10}$ http: //www.kaffe.org/ 
Reading from the device register serial.data, saving the result to the stack

\begin{tabular}{l|lrr|l} 
original bytecode & stack content & & modified bytecode & stack content \\
& & & \\
Gerial $\}$ & & GETFIELD data & $\{$ serial $\}$ \\
Gio port address $\}$ & inval $\}$
\end{tabular}

Writing a value on the stack into the device register serial.data

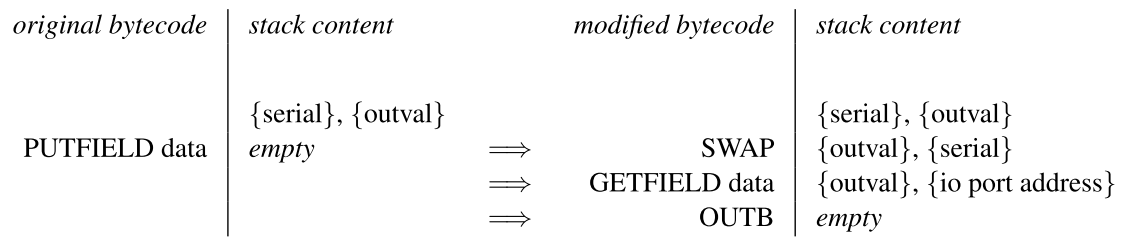

Fig. 24. Translation of bytecode for access to regular fields into bytecode for access to I/O port registers.

We base our access to hardware objects on IR instruction transformations. We introduce two new instructions outb and inb for byte-wide access to I/O ports. Then we employ OVM's instruction rewriting framework to translate accesses to hardware object fields, putfield and getfield instructions, into sequences centered around outb and inb where appropriate. We did not implement word-wide or double-word-wide access modes supported by a x 86 CPU. We discuss how this could be done at the end of this section.

To minimize changes to the OVM code we keep the memory layout of hardware objects as if they were ordinary objects, and store port addresses into the fields representing the respective hardware I/O ports. Explained with the example from Figure 18, the instruction rewriting algorithm proceeds as follows: SerialPort is a subclass of HardwareObject; hence it is a hardware object, and thus accesses to all its public volatile int fields, status and data, are translated to port accesses to I/O addresses stored in those fields.

The translation (Figure 24) is very simple. In case of reads we append our new inb instruction after the corresponding getfield instruction in the IR: getfield will store the $\mathrm{I} / \mathrm{O}$ address on the stack and inb will replace it by a value read from this I/O address.

In case of writes we replace the corresponding putfield instruction by a sequence of swap, getfield, and outb. The swap rotates the two top elements on stack, leaving the hardware object reference on top of the stack and the value to store to the I/O port below it, The getfield replaces the object reference by the corresponding I/O address, and outb writes the value to the I/O port.

The critical part of hardware object creation is to set I/O addresses into hardware object fields. Our approach allows a method to turn off the special handling of hardware objects. In a hardware object factory method accesses to hardware object fields are handled as if they were fields of regular objects; we simply store I/O addresses to the fields.

A method can turn off the special handling of hardware objects with a marker exception mechanism which is a natural solution within OVM. The method declares to throw a PragmaNoHWIORegistersAccess exception. This exception is neither thrown nor caught, but the OVM IR-level rewriter detects the declaration and disables 
940 rewriting accordingly. As the exception extends RuntimeException, it does not need 941 to be declared in interfaces or in code calling factory methods. In Java 1.5, not 942 supported by OVM, a standard substitute to the marker exception would be method 943 annotation.

944 Our solution depends on the representation of byte-wide registers by 16-bit fields 945 to hold the I/O address. However, it could still be extended to support multiple-width 946 accesses to I/O ports (byte, 16 -bit, and 32-bit) as follows: 32-bit I/O registers are rep947 resented by Java long fields, 16-bit I/O registers by Java int fields, and byte-wide I/O 948 registers by Java short fields. The correct access width will be chosen by the IR rewriter 949 based on the field type.

950 4.4.2 Interrupt Handler. Low-level support depends heavily on scheduling and preemp951 tion. For our experiments we chose the uniprocessor x86 OVM configuration with 952 green threads running as a single Linux process. The green threads, delayed I/O opera953 tions, and handlers of asynchronous events, such as POSIX signals, are only scheduled 954 at well-defined points (pollchecks) which are by default at back-branches at bytecode 955 level and indirectly at Java-level blocking calls (I/O operations, synchronization calls, 956 etc). When no thread is ready to run, the OVM scheduler waits for events using the 957 POSIX select call.

958 As OS we use Xenomai RT Linux [Gerum 2004; Xenomai Developers 2008]. Xeno959 mai tasks, which are in fact user-space Linux threads, can run either in the Xenomai 960 primary domain or in the Xenomai secondary domain. In the primary domain they are 961 scheduled by the Xenomai scheduler, isolated from the Linux kernel. In the secondary 962 domain Xenomai tasks behave as regular real-time Linux threads. Tasks can switch to 963 the primary domain at any time, but are automatically switched back to the secondary 964 domain whenever they invoke a Linux system call. A single Linux process can have 965 threads of different types: regular Linux threads, Xenomai primary domain tasks, and 966 Xenomai secondary domain tasks. Primary domain tasks can wait on hardware inter967 rupts with a higher priority than the Linux kernel. The Xenomai API provides the 968 interrupts using the ISR event handler approach and supports virtualization of basic 969 interrupt operations - disabling and enabling a particular interrupt or all local CPU 970 interrupts. These operations have the same semantics as real interrupts, and dis971 abling/enabling a particular one leads to the corresponding operation being performed 972 at the hardware level.

973 Before our extension, OVM ran as a single Linux process with a single (native 974 Linux) thread, a main OVM thread. This native thread implemented Java green 975 threads. To support interrupts we add additional threads to the OVM process: for each 976 interrupt source handled in OVM we dynamically add an interrupt listener thread run977 ning in the Xenomai primary domain. The mechanism that leads to invocation of the 978 Java interrupt handler thread is illustrated in Figure 25.

979 Upon receiving an interrupt, the listener thread marks the pending interrupt in a 980 data structure shared with the main OVM thread. When it reaches a pollcheck, it 981 discovers that an interrupt is pending. The scheduler then immediately wakes-up and 982 schedules the Java green thread that is waiting for the interrupt (IRQ server thread 983 in the figure). To simulate the first-level ISR handler approach, this green thread 984 invokes some handler method. In a non-RTSJ scenario the green thread invokes the 985 run() method of the associated InterruptHandler (see Figure 16). In an RTSJ scenario 986 (not shown in Figure 25), a specialized thread fires an asynchronous event bound to 987 the particular interrupt source. It invokes the fire() method of the respective RTSJ's 988 AsyncEvent. As mentioned in Section 3.3.3 the RTSJ logic of AsyncEventHandler (AEH) 989 registered to this event should be an instance of InterruptHandler in order to allow the 990 interrupt handling code to access basic interrupt handling operations. 


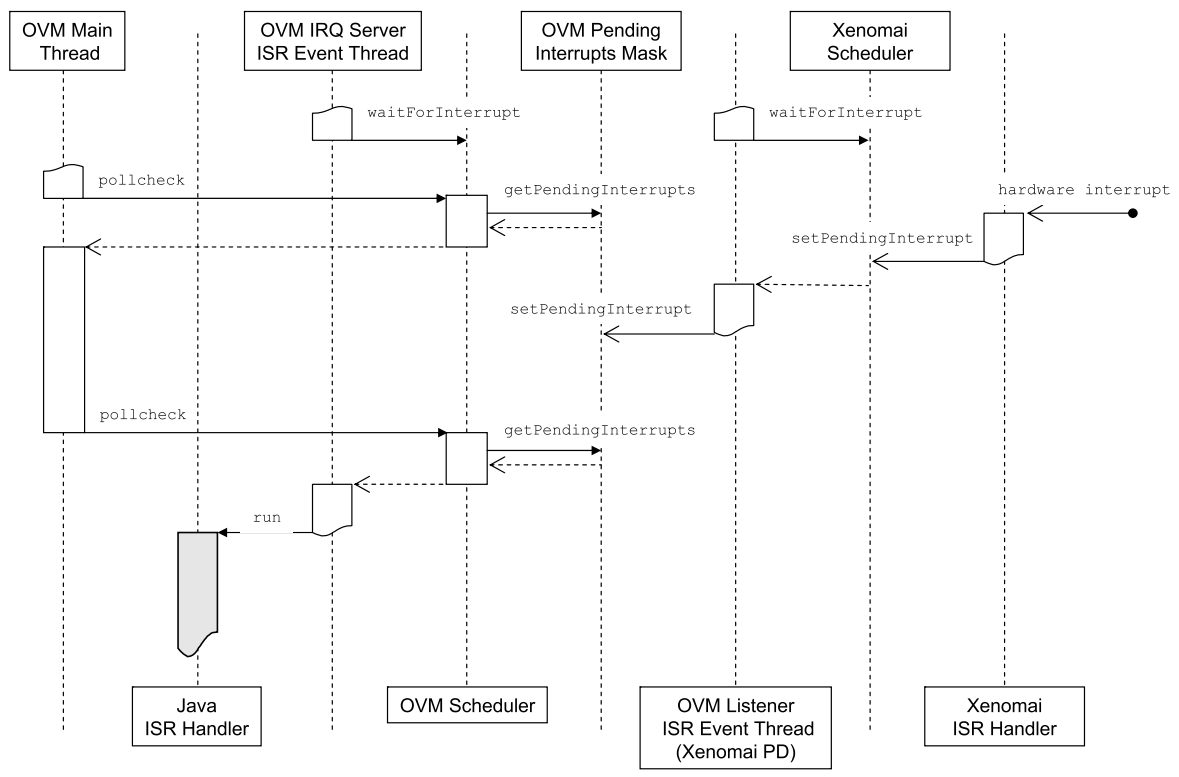

Fig. 25. Invocation of a Java interrupt handler under OVM/Xenomai.

\section{4.5 Summary}

1011 Support for hardware objects (see Section 3.1) and interrupt handling (see Section 3.2) 1012 to all four JVMs relies on common techniques. Accessing device registers through 1013 hardware objects extends the interpretation of the bytecodes putfield and getfield or 1014 redirects the pointer to the object. If these bytecodes are extended to identify the field 1015 being accessed as inside a hardware object, the implementation can use this infor1016 mation. Similarly, the implementation of interrupt handling requires changes to the 1017 bytecodes monitorenter and monitorexit or preinflating a specialized implementation of 1018 a Java monitor. In case of the bytecode extension, the extended codes specify if the 
1019 monitor being acquired belongs to an interrupt handler object. If so, the implemen1020 tation of the actual monitor acquisition must be changed to disable/enable interrupts. 1021 Whether dealing with hardware or interrupt objects, we used the same approach of let1022 ting the hardware object and interrupt handler classes inherit from the superclasses 1023 HardwareObject and InterruptHandler respectively.

1024 For JVMs that need a special treatment of bytecodes putfield and getfield (Sim1025 pleRTJ, Kaffe, and OVM) bytecode rewriting at runtime can be used to avoid the addi1026 tional check of the object type. This is a standard approach (called quick bytecodes in 1027 the first JVM specification) in JVMs to speed-up field access of resolved classes.

1028 Historically, registers of most x86 I/O devices are mapped to a dedicated I/O ad1029 dress space, which is accessed using dedicated instructions: port read and port writes. 1030 Fortunately, both the processor and Linux allow user-space applications running with 1031 administrator privileges to use these instructions and access the ports directly via 1032 iopl, inb, and outb calls. For both the Kaffe and OVM implementations we have imple1033 mented bytecode instructions putfield and getfield accessing hardware object fields by 1034 calls to iopl, inb, and outb.

1035 Linux does not allow user-space applications to handle hardware interrupts. Only 1036 kernel space functionality is allowed to register interrupt handlers. We have overcome 1037 this issue in two different ways.

1038 - For Kaffe we have written a special-purpose kernel module through which the user1039 space application (the Kaffe VM) can register interest in interrupts and get notified 1040 about interrupt occurrence.

1041 - For OVM we have used the Xenomai real-time extension to Linux. Xenomai extends 1042 the Linux kernel to allow for the creation of real-time threads and allows user-space 1043 code to wait for interrupt occurrences.

1044 Both these work-arounds allow an incremental transition of the JVMs and the re1045 lated development libraries into a direct (bare metal) execution environment. In that 1046 case the work-arounds would no longer be needed.

1047 If a compiling JVM is used (either as JIT or ahead-of-time) the compiler needs to be 1048 aware of the special treatment of hardware objects and monitors on interrupt handlers. 1049 One issue which we did not face in our implementations was the alignment of object 1050 fields. When device registers are represented by differently sized integer fields, the 1051 compiler needs to pack the data structure.

1052 The restrictions within an interrupt handler are JVM-dependent. If an interrupt1053 ible, real-time GC is used (as in OVM and JOP) objects can be allocated in the handler 1054 and the object graph may be changed. For a JVM with a stop-the-world GC (SimpleRTJ 1055 and Kaffe) allocations are not allowed because the handler can interrupt the GC.

\section{5. EVALUATION AND CONCLUSION}

1057 Having implemented the Java HAL on four different JVMs we evaluate it on a sev1058 eral test applications, including a tiny Web server, and measure the performance of 1059 hardware accesses via hardware objects and the latency of Java interrupt handlers.

\subsection{Qualitative Observations}

1061 For first tests we implemented a serial port driver with hardware objects and interrupt 1062 handlers. As the structure of the device registers is exactly the same on a PC, the 1063 platform for SimpleRTJ, and JOP, we were able to use the exact same definition of the 1064 hardware object SerialPort and the test programs on all four systems.

1065 Using the serial device we run an embedded TCP/IP stack, implemented completely 1066 in Java, over a SLIP connection. The TCP/IP stack contains a tiny Web server and we 
1067 serve Web pages with a Java-only solution similar to the one shown in the Introduction 1068 in Figure 6. The TCP/IP stack, the tiny Web server, and the hardware object for the 1069 serial port are the same for all platforms. The only difference is in the hardware object 1070 creation with the platform-dependent factory implementations. The Web server uses 1071 hardware objects and polling to access the serial device.

1072 5.1.1 A Serial Driver in Java. For testing the interrupt handling infrastructure in OVM 1073 we implemented a serial interrupt-based driver in Java and a demo application that 1074 sends back the data received through a serial interface. The driver part of the applica1075 tion is a full-duplex driver with support for hardware flow control and with detection 1076 of various error states reported by the hardware. The driver uses two circular buffers, 1077 one for receiving and the other for sending. The user part of the driver implements 1078 blocking getChar and putChar calls, which have (short) critical sections protected by 1079 the interrupt-disabling monitor. To reduce latencies the getChar call sets the DSR flag 1080 to immediately allow receiving more data and the putChar, after putting the charac1081 ter into the sending buffer, initiates immediately the sending, if this is not currently 1082 being done already by the interrupt machinery. The driver supports serial ports with 1083 a FIFO buffer. The user part of the demo application implements the loop-back using 1084 getChar and putChar. The user part is a RTSJ AsyncEventHandler which is fired when 1085 a new character is received. From a Java perspective this is a second-level interrupt 1086 handler, invoked after the corresponding serial event is fired from the first-level han1087 dler. To test the API described in the article we implemented two versions that differ 1088 in how the first-level handler is bound to the interrupt: (a) a RTSJ-style version where 1089 the first-level handler is also a RTSJ event handler bound using bindTo to the JVM 1090 provided first-level serial event, and (b) a non-RTSJ-style version where the first-level 1091 handler is registered using a InterruptHandler.register call. We have stress-tested the 1092 demo application and the underlying modified OVM infrastructure by sending large 1093 files to it through the serial interface and checked that they were returned intact.

1094 5.1.2 The HAL in Daily Use. The original idea for hardware objects evolved during de1095 velopment of low-level software on the JOP platform. The abstraction with read and 1096 write functions and using constants to represent I/O addresses just felt wrong with 1097 Java. Currently hardware objects are used all over in different projects with JOP. 1098 Old code has been refactored to some extent, but new low-level code uses only hard1099 ware objects. By now low-level I/O is integrated into the language, for example, auto1100 completion in the Eclipse IDE makes it easy to access the factory methods and fields 1101 in the hardware object.

1102 For experiments with an on-chip memory for thread-local scope caching [Wellings 1103 and Schoeberl 2009] in the context of a chip-multiprocessor version of JOP, the hard1104 ware array abstraction greatly simplified the task. The on-chip memory is mapped to 1105 a hardware array and the RTSJ-based scoped memory uses it. Creation of an object 1106 within this special scope is implemented in Java and is safe because the array bounds 1107 checks are performed by the JVM.

1108 5.1.3 JNI vs. Hardware Objects. JNI provides a way to access the hardware without 1109 changing the code of the JVM. Nevertheless, with a lack of commonly agreed API, 1110 using it for each application would be redundant and error prone. It would also add 1111 dependencies to the application: hardware platform and the operating system (the $\mathrm{C}$ 1112 API for accessing the hardware is not standardized). The build process is complicated 1113 by adding $\mathrm{C}$ code to it as well. Moreover, the system needs to support shared libraries, 1114 which is not always the case for embedded operating systems (an example is RTEMS, 1115 used by ESA). 
1116 In addition, JNI is typically too heavyweight to implement trivial calls such as 1117 port or memory access efficiently (no GC interaction, no pointers, no threads inter1118 action, no blocking). Even JVMs that implement JNI usually have some other inter1119 nal lightweight native interface which is the natural choice for hardware access. This 1120 leads us back to a Java HAL as illustrated here.

1121 5.1.4 OVM-Specific Experience. Before the addition of hardware objects, OVM did not 1122 allow hardware access because it did not and does not have JNI or any other native 1123 interface for user Java code. OVM has a simplified native interface for the virtual 1124 machine code which indeed we used when implementing the hardware objects. This 1125 native interface can as well be used to modify OVM to implement user-level access to 1126 hardware via regular method calls. We have done this to implement a benchmark to 1127 measure HWO/native overheads (later in this section). As far as simple port access 1128 is concerned, none of the solutions is strictly better from the point of the JVM: the 1129 bytecode manipulation to implement hardware objects was easy, as well as adding code 1130 to propagate native port I/O calls to user code. Thanks to ahead-of-time compilation 1131 and the simplicity of the native interface, the access overhead is the same.

1132 The OVM compiler is fortunately not "too smart" so it does not get in the way of 1133 supporting hardware objects: if a field is declared volatile side-effects of reading of 1134 that field are not a problem for any part of the system.

1135 The API for interrupt handling added to OVM allows full control over interrupts, 1136 typically available only to the operating system. The serial port test application has 1137 shown that, at least for a simple device; it really allows us to write a driver. An in1138 teresting feature of this configuration is that OVM runs in user space and therefore it 1139 greatly simplifies development and debugging of Java-only device drivers for embed1140 ded platforms.

\section{5.2 Performance}

1142 Our main objective for hardware objects is a clean object-oriented interface to hard1143 ware devices. Performance of device register access is an important goal for relatively 1144 slow embedded processors; thus we focus on that in the following. It matters less on 1145 general-purpose processors where the slow I/O bus essentially limits the access time.

$1146 \quad$ 5.2.1 Measurement Methodology. Execution time measurement of single instructions 1147 is only possible on simple in-order pipelines when a cycle counter is available. On 1148 a modern superscalar architecture, where hundreds of instructions are in flight each 1149 clock cycle, direct execution time measurement becomes impossible. Therefore, we 1150 performed a bandwidth-based measurement. We measure how many I/O instructions 1151 per second can be executed in a tight loop. The benchmark program is self-adapting 1152 and increases the loop count exponentially until the measurement runs for more than 1153 one second and the iterations per second are reported. To compensate for the loop 1154 overhead we perform an overhead measurement of the loop and subtract that overhead 1155 from the I/O measurement. The I/O bandwidth $b$ is obtained as follows.

$$
b=\frac{c n t}{t_{\text {test }}-t_{\text {ovhd }}}
$$

1156 Figure 26 shows the measurement loop for the read operation in method test() and 1157 the overhead loop in method overhead(). In the comment above the method the byte1158 codes of the loop kernel are shown. We can see that the difference between the two 1159 loops is the single bytecode getfield that performs the read request.

$1160 \quad$ 5.2.2 Execution Time. In Table III we compare the access time with native functions 1161 to the access via hardware objects. The execution time is given in clock cycles. We 


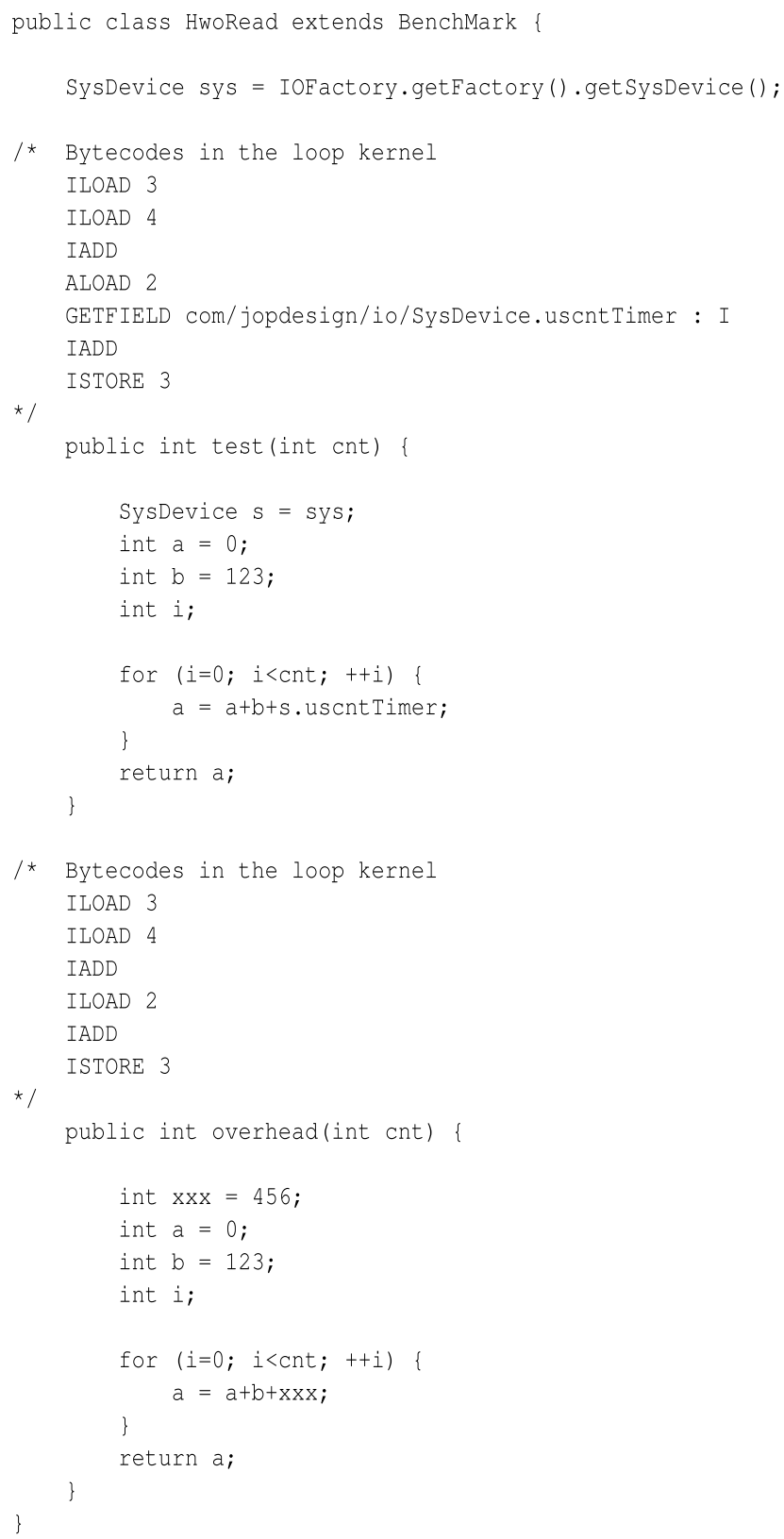

Fig. 26. Benchmark for the read operation measurement.

1162 scale the measured I/O bandwidth $b$ with the clock frequency $f$ of the system under 1163 test by $n=\frac{f}{b}$.

1164 We have run the measurements on a $100 \mathrm{MHz}$ version of JOP. As JOP is a sim1165 ple pipeline, we can also measure short bytecode instruction sequences with the cycle 1166 counter. Those measurements provided the exact same values as the ones given by our 1167 benchmark, such that they validated our approach. 
Table III. Access Time to a Device Register in Clock Cycles

\begin{tabular}{lrcccccrr}
\hline & \multicolumn{2}{c}{ JOP } & \multicolumn{2}{c}{ OVM } & \multicolumn{2}{c}{ SimpleRTJ } & \multicolumn{2}{c}{ Kaffe } \\
& read & write & read & write & read & write & read & write \\
\hline native & 5 & 6 & 5517 & 5393 & 2588 & 1123 & 11841 & 11511 \\
HW Object & 13 & 15 & 5506 & 5335 & 3956 & 3418 & 9571 & 9394 \\
\hline
\end{tabular}

1168 On JOP the native access is faster than using hardware objects because a native ac1169 cess is a special bytecode and not a native function call. The special bytecode accesses 1170 memory directly where the bytecodes putfield and getfield perform a null pointer check 1171 and indirection through the handle for the field access. Despite the slower I/O ac1172 cess via hardware objects on JOP, the access is fast enough for all currently available 1173 devices. Therefore, we will change all device drivers to use hardware objects. The 1174 measurement for OVM was run on a Dell Precision 380 (Intel Pentium 4, $3.8 \mathrm{GHz}$, 1175 3G RAM, 2M 8-way set associative L2 cache) with Linux (Ubuntu 7.10, Linux 2.6.24.3 1176 with Xenomai-RT patch). OVM was compiled without Xenomai support and the gen1177 erated virtual machine was compiled with all optimizations enabled. As I/O port we 1178 used the printer port. Access to the I/O port via a hardware object is just slightly faster 1179 than access via native methods. This was expected as the slow I/O bus dominates the 1180 access time. On the SimpleRTJ JVM the native access is faster than access to hard1181 ware objects. The reason is that the JVM does not implement JNI, but has its own 1182 proprietary, more efficient way to invoke native methods. It is done in a prelinking 1183 phase where the invokestatic bytecode is instrumented with information to allow an 1184 immediate invocation of the target native function. On the other hand, using hard1185 ware objects needs a field lookup that is more time consuming than invoking a static 1186 method. With bytecode-level optimization at class load time it would be possible to 1187 avoid the expensive field lookup.

1188 We measured the I/O performance with Kaffe on an Intel Core 2 Duo T7300, $11892.00 \mathrm{GHz}$ with Linux 2.6.24 (Fedora Core 8). We used access to the serial port for 1190 the measurement. On the interpreting Kaffe JVM we notice a difference between the 1191 native access and hardware object access. Hardware objects are around $20 \%$ faster.

1192 5.2.3 Summary. For practical purposes the overhead on using hardware objects is 1193 insignificant. In some cases there may even be an improvement in performance. The 1194 benefits in terms of safe and structured code should make this a very attractive option 1195 for Java developers.

11965.3 Interrupt Handler Latency

1197 5.3.1 Latency on JOP. To measure interrupt latency on JOP we use a periodic thread 1198 and an interrupt handler. The periodic thread records the value of the cycle counter 1199 and triggers the interrupt. In the handler the counter is read again and the differ1200 ence between the two is the measured interrupt latency. A plain interrupt handler 1201 as Runnable takes a constant 234 clock cycles (or $2.3 \mu$ s for a $100 \mathrm{MHz} \mathrm{JOP}$ system) 1202 between the interrupt occurrence and the execution of the first bytecode in the han1203 dler. This quite large time is the result of two method invocations for the interrupt 1204 handling: (1) invocation of the system method interrupt() and (2) invocation of the ac1205 tual handler. For more time-critical interrupts the handler code can be integrated in 1206 the system method. In that case the latency drops down to $0.78 \mu \mathrm{s}$. For very low1207 latency interrupts the interrupt controller can be changed to emit different bytecodes 1208 depending on the interrupt number, then we avoid the dispatch in software and can 1209 implement the interrupt handler in microcode. 
Table IV. Interrupt (and polling) Latencies in Microseconds

\begin{tabular}{lcccc}
\hline & Median $(\mu \mathrm{s})$ & 3rd Quartile $(\mu \mathrm{s})$ & 95\% Quantile $(\mu \mathrm{s})$ & Maximum $(\mu \mathrm{s})$ \\
\hline Polling & 3 & 3 & 3 & 8 \\
Kernel & 14 & 16 & 16 & 21 \\
Hard & 14 & 16 & 16 & 21 \\
User & 17 & 19 & 19 & 24 \\
Ovm & 59 & 59 & 61 & 203 \\
\hline
\end{tabular}

1210 We have integrated the two-level interrupt handling at the application level. We 1211 set up two threads: one periodic thread, that triggers the interrupt, and a higher1212 priority event thread that acts as second-level interrupt handler and performs the 1213 handler work. The first-level handler just invokes fire() for this second-level handler 1214 and returns. The second-level handler gets scheduled according to the priority. With 1215 this setup the interrupt handling latency is $33 \mu \mathrm{s}$. We verified this time by measuring 1216 the time between fire of the software event and the execution of the first instruction 1217 in the handler directly from the periodic thread. This took $29 \mu \mathrm{s}$ and is the overhead 1218 due to the scheduler. The value is consistent with the measurements in Schoeberl and 1219 Vitek [2007]. There we measured a minimum useful period of $50 \mu \mathrm{s}$ for a high-priority 1220 periodic task.

1221 The runtime environment of JOP contains a concurrent real-time GC [Schoeber] 1222 and Vitek 2007]. The GC can be interrupted at a very fine granularity. During sections 1223 that are not preemptive (data structure manipulation for a new and write-barriers on 1224 a reference field write) interrupts are simply turned off. The copy of objects and ar1225 rays during the compaction phase can be interrupted by a thread or interrupt handler 1226 [Schoeberl and Puffitsch 2008]. Therefore, the maximum blocking time is in the atomic 1227 section of the thread scheduler and not in the GC.

1228 5.3.2 Latency on OVM/Xenomai. For measuring OVM/Xenomai interrupt latencies, we 1229 have extended an existing interrupt latency benchmark, written by Jan Kiszka from 1230 the Xenomai team [Xenomai Developers 2008]. The benchmark uses two machines 1231 connected over a serial line. The log machine, running a regular Linux kernel, toggles 1232 the RTS state of the serial line and measures the time it takes for the target machine 1233 to toggle it back.

1234 To minimize measuring overhead the log machine uses only polling and disables lo1235 cal CPU interrupts while measuring. Individual measurements are stored in memory 1236 and dumped at shutdown so that they can be analyzed offline. We have made 400,000 1237 measurements in each experiment, reporting only the last 100,000 (this was to warm1238 up the benchmark, including memory storage for the results). The log machine toggles 1239 the RTS state regularly with a given period.

1240 We have tested 5 versions of the benchmark on the target machine: a polling version 1241 written in $\mathrm{C}$ (polling), a kernel-space interrupt handler in C/Xenomai running out of 1242 control of the Linux scheduler (kernel), a hard-realtime kernel-space interrupt handler 1243 running out of control of both the Xenomai scheduler and the Linux scheduler (hard), 1244 a user-space interrupt handler written in C/Xenomai (user), and finally an interrupt 1245 handler written in Java/OVM/Xenomai (ovm).

1246 The results are shown in Table IV. The median latency is $3 \mu \mathrm{s}$ for polling, $14 \mu \mathrm{s}$ for 1247 both kernel-space handlers (hard and kernel), $17 \mu$ s for user-space C handler (user), 1248 and $59 \mu \mathrm{s}$ for Java handler in OVM (ovm). Note that the table shows that the overhead 1249 of using interrupts over polling is larger than the overhead of handling interrupts 1250 in user space over kernel space. The maximum latency of OVM was $203 \mu \mathrm{s}$, due to 
1251 infrequent pauses. Their frequency is so low that the measured $95 \%$ quantile is only $125261 \mu \mathrm{s}$.

1253 The experiment was run on Dell Precision 380 (Intel Pentium $43.8 \mathrm{GHz}, 3 \mathrm{G}$ RAM, 1254 2M 8-way set associative L2 cache) with Linux (Ubuntu 7.10, Linux 2.6.24.3 with 1255 Xenomai-RT patch). As Xenomai is still under active development we had to use 1256 Xenomai work-arounds and bugfixes, mostly provided by Xenomai developers, to make 1257 OVM on Xenomai work.

1258 5.3.3 Summary. The overhead for implementing interrupt handlers is very accept1259 able since interrupts are used to signal relatively infrequently occurring events like 1260 end of transmission, loss of carrier, etc. With a reasonable work division between 1261 first-level and second-level handlers, the proposal does not introduce dramatic block1262 ing terms in a real-time schedulability analysis, and thus it is suitable for embedded 1263 systems.

\subsection{Discussion}

1265 5.4.1 Safety Aspects. Hardware objects map object fields to the device registers. When 1266 the class that represents a device is correct, access to it is safe; it is not possible to read 1267 from or write to an arbitrary memory address. A memory area represented by an array 1268 is protected by Java's array bounds check.

1269 5.4.2 Portability. It is obvious that hardware objects are platform-dependent; after all 1270 the idea is to have an interface to the bare metal. Nevertheless, hardware objects give 1271 device manufacturers an opportunity to supply supporting factory implementations 1272 that fit into Java's object-oriented framework and thus cater for developers of embed1273 ded software. If the same device is used on different platforms, the hardware object is 1274 portable. Therefore, standard hardware objects can evolve.

1275 5.4.3 Compatibility with the RTSJ Standard. As shown for the OVM implementation, the 1276 proposed HAL is compatible with the RTSJ standard. We consider it to be a very im1277 portant point since many existing systems have been developed using such platforms 1278 or subsets thereof. In further development of such applications existing and future in1279 terfacing to devices may be refactored using the proposed HAL. It will make the code 1280 safer and more structured and may assist in possible ports to new platforms.

\subsection{Perspective}

1282 The many examples in the text show that we achieved a representation of the hard1283 ware close to being platform-independent. Also, they show that it is possible to imple1284 ment system-level functionality in Java. As future work we consider to add devices 1285 drivers for common devices such as network interfaces ${ }^{11}$ and hard disc controllers. 1286 On top of these drivers we will implement a file system and other typical OS-related 1287 services towards our final goal of a Java-only system.

1288 An interesting question is whether a common set of standard hardware objects is 1289 definable. The SerialPort was a lucky example. Although the internals of the JVMs and 1290 the hardware were different one compatible hardware object worked on all platforms. 1291 It should be feasible that a chip manufacturer provides, beside the data sheet that 1292 describes the registers, a Java class for the register definitions of that chip. This defi1293 nition can be reused in all systems that use that chip, independent of the JVM or OS.

1294 Another interesting idea is to define the interaction between the GC and hardware 1295 objects. We stated that the GC should not collect hardware objects. If we relax this

\footnotetext{
${ }^{11}$ A device driver for a CS8900-based network chip is already part of the Java TCP/IP stack.
} 
1296 restriction we can redefine the semantics of collecting an object: on running the 1297 finalizer for a hardware object the device can be put into sleep mode.

\section{ACKNOWLEDGMENTS}

1299 We wish to thank Andy Wellings for his insightful comments on an earlier version of the article. We also 1300 thank the reviewers for their detailed comments that helped to enhance the original submission.

\section{REFERENCES}

1302 AJILE. 2000. aj-100 real-time low power Java processor. Preliminary data sheet.

1303 Armbruster, A., Baker, J., Cunei, A., Flack, C., Holmes, D., Pizlo, F., Pla, E., Prochazka, M., 1304 AND ViteK, J. 2007. A real-time Java virtual machine with applications in avionics. Trans. Embed. 1305 Comput. Sys. 7, 1, 1-49.

1306 Bacon, D. F., Cheng, P., And Rajan, V. T. 2003. A real-time garbage collector with low overhead and 1307 consistent utilization. In Proceedings of the 30th ACM SIGPLAN-SIGACT Symposium on Principles of 1308 Programming Languages (POPL'03). ACM Press, New York, 285-298.

1309 Bollella, G., Gosling, J., Brosgol, B., Dibble, P., Furr, S., And Turnbull, M. 2000. The Real-Time 1310 Specification for Java. Java Series. Addison-Wesley.

1311 Burns, A. AND Wellings, A. J. 2001. Real-Time Systems and Programming Languages: ADA 95, Real-

1312 Time Java, and Real-Time POSIX 3rd Ed. Addison-Wesley Longman Publishing.

1313 CASKA, J. accessed 2009. micro [ $\mu$ ] virtual-machine. http://muvium. com/.

1314 Chou, A., Yang, J., Chelf, B., Hallem, S., And Engler, D. 2001. An empirical study of operating 1315 systems errors. SIGOPS Oper. Syst. Rev. 35, 5, 73-88.

1316 Felser, M., Golm, M., Wawersich, C., And Kleinöder, J. 2002. The JX operating system. In Proceed1317 ings of the USENIX Annual Technical Conference. 45-58.

1318 Gamma, E., Helm, R., Johnson, R., And Vlissides, J. M. 1994. Design Patterns: Elements of Reusable 1319 Object-Oriented Software. Addison Wesley Professional.

1320 Gay, D., Levis, P., von Behren, R., Welsh, M., Brewer, E., AND Culler, D. 2003. The nesC language: A holistic approach to networked embedded systems. In Proceedings of the ACM SIGPLAN Conference on Programming Language Design and Implementation (PLDI'O3). ACM Press, New York, 1-11.

Gerum, P. 2004. Xenomai - Implementing a RTOS emulation framework on GNU/Linux. http://www .xenomai.org/documentation/branches/v2.4.x/pdf/xenomai.pdf.

Group, T. C. 2008. Trusted computing. https://www.trustedcomputinggroup.org/.

Hansen, P. B. 1977. The Architecture of Concurrent Programs. Prentice-Hall Series in Automatic Computing. Prentice-Hall.

Hennessy, J. And Patterson, D. 2002. Computer Architecture: A Quantitative Approach 3rd Ed. Morgan Kaufmann Publishers, Palo Alto, CA.

Henties, T., Hunt, J. J., Locke, D., Nilsen, K., Schoeberl, M., And Vitek, J. 2009. Java for safetycritical applications. In Proceedings of the 2nd International Workshop on the Certification of SafetyCritical Software Controlled Systems (SafeCert'09).

Hill, J., Szewczyk, R., Woo, A., Hollar, S., Culler, D. E., ANd Pister, K. S. J. 2000. System architecture directions for networked sensors. In Proceedings of the 9th International Conference on Architectural Support for Programming Languages and Operating Systems (ASPLOS IX). 93-104.

Hunt, G., Larus, J. R., Abadi, M., Aiken, M., Barham, P., Fahndrich, M., Hawblitzel, C., Hodson, O., Levi, S., MurPhy, N., SteensgaArd, B., Tarditi, D., Wobber, T., AND Zill, B. D. 2005. An overview of the singularity project. Tech. rep. MSR-TR-2005-135, Microsoft Research (MSR).

Korsholm, S., Schoeberl, M., AND RAvn, A. P. 2008. Interrupt handlers in Java. In Proceedings of the 11th IEEE International Symposium on Object/Component/Service-Oriented Real-Time Distributed Computing (ISORC'08). IEEE Computer Society.

Krall, A. AND Grafl, R. 1997. CACAO - A 64 bit JavaVM just-in-time compiler. In Proceedings of the Workshop on Java for Science and Engineering Computation (PPoPP'97), G. C. Fox and W. Li Eds. ACM.

Kreuzinger, J., Brinkschulte, U., Pfeffer, M., Uhrig, S., AND Ungerer, T. 2003. Real-Time eventhandling and scheduling on a multithreaded Java microcontroller. Microprocess. Microsyst. 27, 1, 19-31.

Lindholm, T. And Yellin, F. 1999. The Java Virtual Machine Specification 2nd Ed. Addison-Wesley, Reading, MA.

1348 Lohmeier, S. 2005. Jini on the Jnode Java os. http://monochromata.de/jnodejini.html. 
1349 Phipps, G. 1999. Comparing observed bug and productivity rates for java and c++. Softw. Pract. Exper. 29, 4, $1350 \quad 345-358$.

1351 Ravn, A. P. 1980. Device monitors. IEEE Trans. Softw. Engin. 6, 1, 49-53.

RTJ Computing. 2000. SimpleRTJ a small footprint Java VM for embedded and consumer devices. http://www.rtjcom.com/.

1354 SchoeberL, M. 2005. Jop: A java optimized processor for embedded real-time systems. Ph.D. thesis, Vienna 1355 University of Technology.

1356 Schoeberl, M. 2006. Real-Time garbage collection for Java. In Proceedings of the 9th IEEE International 1357 Symposium on Object and Component-Oriented Real-Time Distributed Computing (ISORC'06). IEEE, $1358 \quad 424-432$

1359 Schoeberl, M. 2008. A Java processor architecture for embedded real-time systems. J. Syst. Archit. 54/1-2, $1360 \quad 265-286$

1361 Schoeberl, M. AND Vitek, J. 2007. Garbage collection for safety critical Java. In Proceedings of the 5th

1380 Wilkinson, T. 1996. Kaffe - A virtual machine to run java code. http://www.kaffe.org.

1381 Wirth, N. 1977. Design and implementation of modula. Softw. Pract. Exper. 7, 3-84.

1382 Wirth, N. 1982. Programming in Modula-2. Springer.

1383 Xenomai Developers. 2008. Xenomai: Real-Time framework for Linux. http://www. xenomai.org.

1384 Received August 2008; revised July 2009; accepted February 2010 